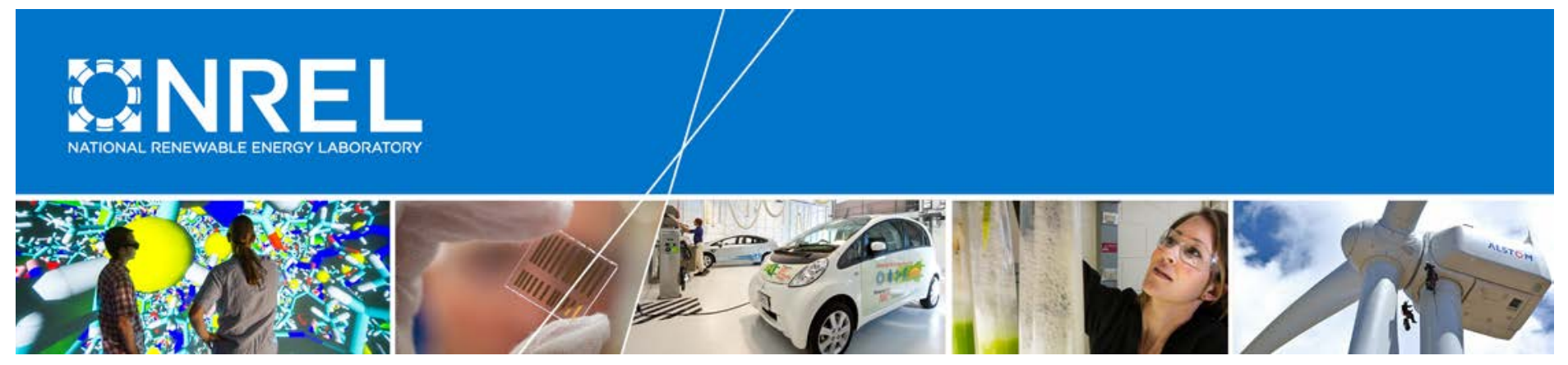

\title{
Validation of Tendril TrueHome Using Software-to-Software Comparison
}

Jeff Maguire and Scott Horowitz National Renewable Energy Laboratory

Nathan Moore and Patrick Sullivan Tendril

NREL is a national laboratory of the U.S. Department of Energy Office of Energy Efficiency \& Renewable Energy Operated by the Alliance for Sustainable Energy, LLC

This report is available at no cost from the National Renewable Energy Laboratory (NREL) at www.nrel.gov/publications.

Technical Report

NREL/TP-5500-70116

September 2017

Contract No. DE-AC36-08G028308 


\section{Validation of Tendril TrueHome Using Software-to-Software Comparison}

Jeff Maguire and Scott Horowitz National Renewable Energy Laboratory

Nathan Moore and Patrick Sullivan Tendril

Prepared under Task No. WTNE.1000
NREL is a national laboratory of the U.S. Department of Energy Office of Energy Efficiency \& Renewable Energy Operated by the Alliance for Sustainable Energy, LLC

This report is available at no cost from the National Renewable Energy Laboratory (NREL) at www.nrel.gov/publications.

\section{Technical Report}

NREL/TP-5500-70116

September 2017

Contract No. DE-AC36-08G028308
National Renewable Energy Laboratory 15013 Denver West Parkway 303-275-3000 • www.nrel.gov 


\section{NOTICE}

This report was prepared as an account of work sponsored by an agency of the United States government. Neither the United States government nor any agency thereof, nor any of their employees, makes any warranty, express or implied, or assumes any legal liability or responsibility for the accuracy, completeness, or usefulness of any information, apparatus, product, or process disclosed, or represents that its use would not infringe privately owned rights. Reference herein to any specific commercial product, process, or service by trade name, trademark, manufacturer, or otherwise does not necessarily constitute or imply its endorsement, recommendation, or favoring by the United States government or any agency thereof. The views and opinions of authors expressed herein do not necessarily state or reflect those of the United States government or any agency thereof.

This report is available at no cost from the National Renewable Energy Laboratory (NREL) at www.nrel.gov/publications.

Available electronically at SciTech Connect http:/www.osti.gov/scitech

Available for a processing fee to U.S. Department of Energy and its contractors, in paper, from:

U.S. Department of Energy

Office of Scientific and Technical Information

P.O. Box 62

Oak Ridge, TN 37831-0062

OSTI http://www.osti.gov

Phone: 865.576.8401

Fax: 865.576.5728

Email: reports@osti.gov

Available for sale to the public, in paper, from:

U.S. Department of Commerce

National Technical Information Service

5301 Shawnee Road

Alexandria, VA 22312

NTIS http://www.ntis.gov

Phone: 800.553 .6847 or 703.605 .6000

Fax: 703.605.6900

Email: orders@ntis.gov 


\section{Acknowledgments}

This work was funded by Tendril and makes use of methods developed previously under funding from U.S. Department of Energy Building Technologies Office. The authors would like to thank Mark Gately of Tendril and Dane Christensen of the National Renewable Energy Laboratory for their oversight and management of this project.

\section{Suggested Citation}

Maguire, J.; Horowitz, S.; Moore, N.; Sullivan, P. (2017). Validation of Tendril TrueHome Using Software-to-Software Comparison. Golden, CO; National Renewable Energy Laboratory.

NREL/SR-5500-70116. http://www.nrel.gov/docs/fy17osti/70116.pdf 


\section{Executive Summary}

This study performed comparative evaluation of EnergyPlus ${ }^{\mathrm{TM}}$ version 8.6 (Department of Energy 2016) and Tendril TrueHome, a physics-based home energy simulation model (Tendril 2014) to identify differences in energy consumption predictions between the two programs and resolve discrepancies between them. EnergyPlus is considered a benchmark, best-in-class software tool for building energy simulation. This exercise sought to improve both software tools through additional evaluation/scrutiny.

This study was performed using BEopt, a residential building front-end developed by the National Renewable Energy Laboratory that leverages existing simulation engines such as EnergyPlus. By connecting BEopt to Tendril's TrueHome model, side by side comparisons between TrueHome and EnergyPlus predictions could be quickly performed. This project involved connecting as many Tendril TrueHome technologies as possible to BEopt, followed by comparing energy predictions to EnergyPlus. The comparative evaluation capability of BEopt, known as the "BEopt Test Suite," allows comparison of simulation results specific to hundreds of different building characteristics one-at-a-time (Horowitz et al. 2016). This capability has been useful in identifying and correcting bugs and errors in several building energy models including SEEM, DOE-2 and EnergyPlus. During the current study a variety of differences in the predictions made by the models were identified. Several discrepancies in methodology, some of which affected the results across many technologies, were identified and fixed as a part of this study. In addition, some key differences in modeling assumptions that significantly impacted the results for some technologies were identified, with subsequent adjustments made. After performing corrections, Tendril TrueHome showed good agreement with EnergyPlus for the test cases considered here as shown in Figure ES1b. The average discrepancy in diagnostic buildings was $13 \%$ in the final results. Additionally, typical buildings representative of current construction practices had an average difference of less than $10 \%$. Some technologies were not explored due to limitations in comparing the engines including ducts, crawlspaces, and basements. In addition to improvements in the Tendril Building Model, a modeling error was discovered in the boiler model used in BEopt, which has since been fixed. 


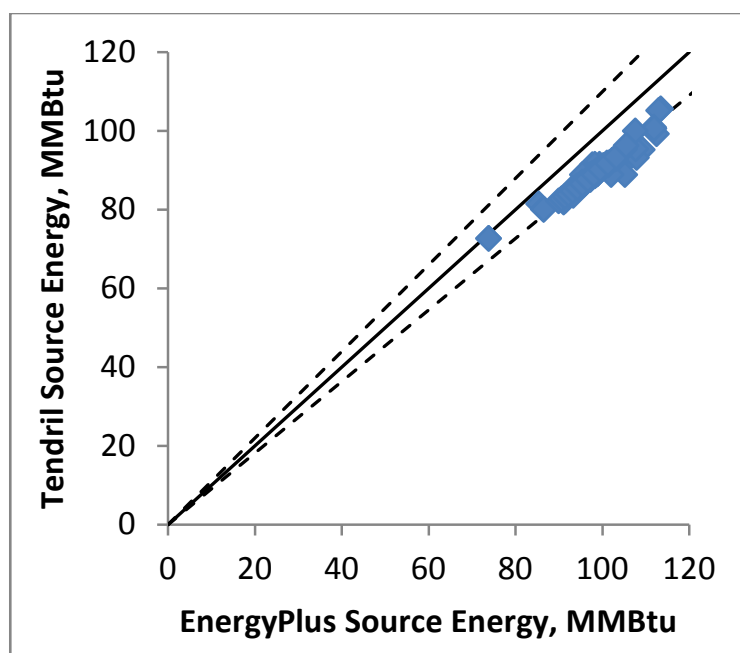

(a)

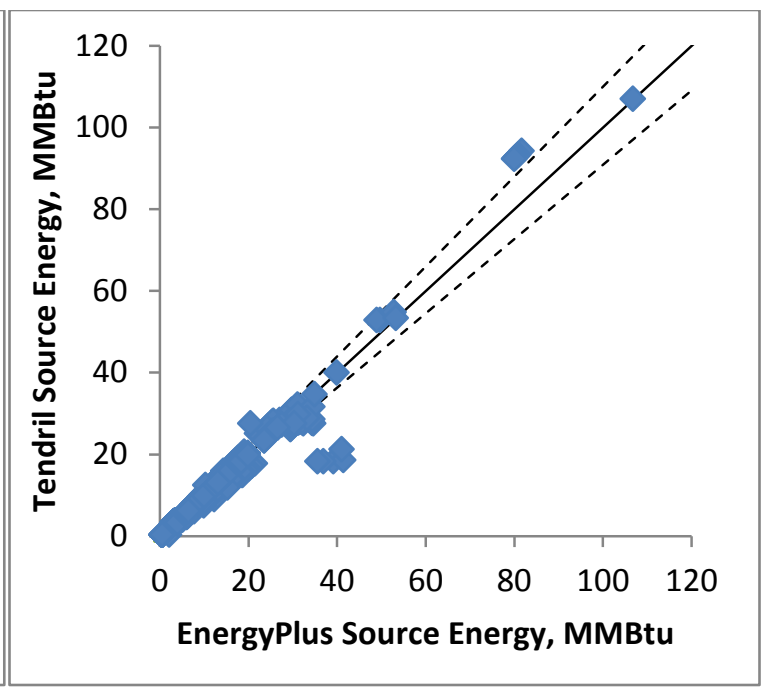

(b)

Figure ES1. Final test suite results for a typical (a) and diagnostic (b) building in Atlanta, GA; dashed lines indicate $\pm 10 \%$ 


\section{Acronyms}

ANSI American National Standards Institute

BEopt Building Energy Optimization Tool

DSE distribution system efficiency

HPWH heat pump water heater

HVAC heating, ventilating, and air conditioning

NREL National Renewable Energy Laboratory

SEEM Simple Energy and Enthalpy Model

SEER seasonal energy efficiency ratio

SHR sensible heat ratio 


\section{Table of Contents}

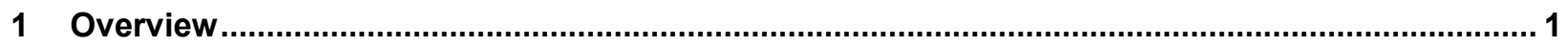

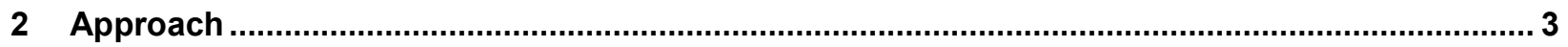

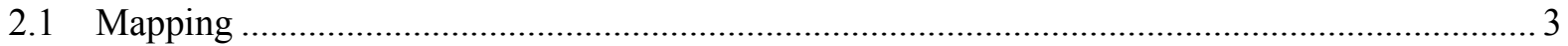

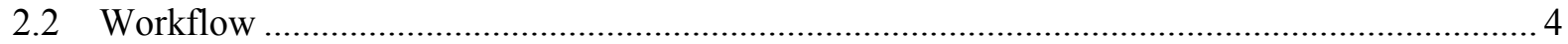

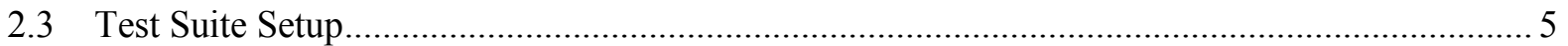

2.4 Versions of EnergyPlus and Tendril TrueHome Used ..................................................... 8

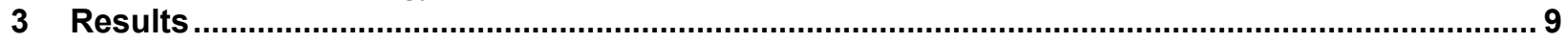

3.1 Overall Energy Consumption Comparison ............................................................ 9

3.2 Discrepancies Corrected ........................................................................................ 11

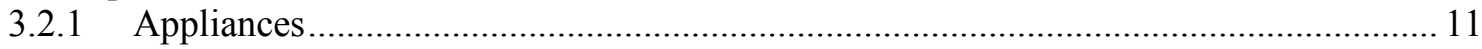

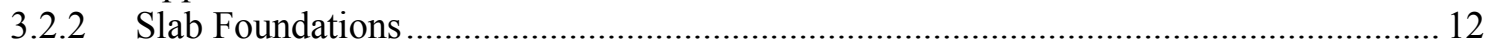

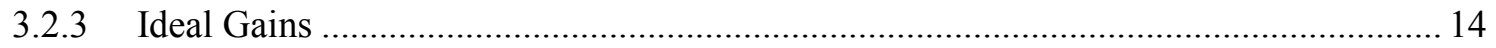

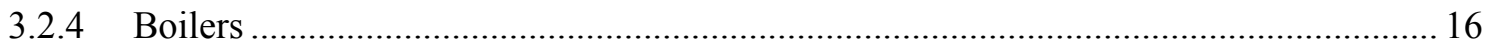

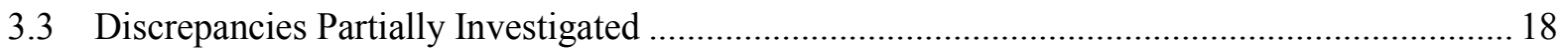

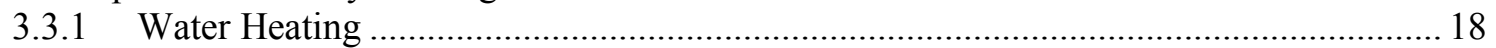

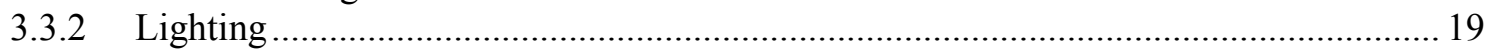

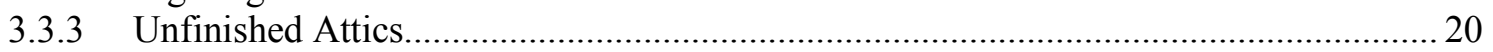

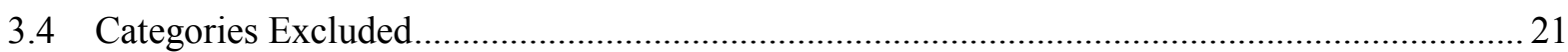

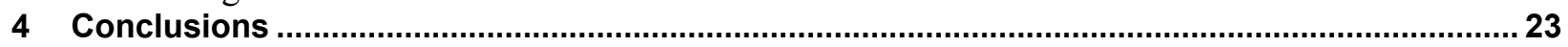

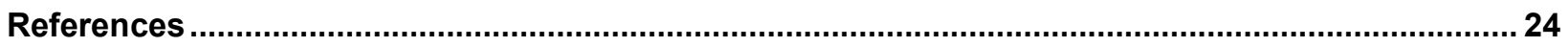

Appendix A: Mapping Table ....................................................................................................... 25

Appendix B: BEopt TrueHome/EnergyPlus Test Suite Coverage.................................................. 29 


\section{List of Figures}

Figure ES1. Final test suite results for a typical (a) and diagnostic (b) building in Atlanta, GA; dashed lines indicate $\pm 10 \%$

Figure 1. Schematic of BEopt use cases. Top shows BEopt performing optimization involving multiple EnergyPlus simulations. Bottom shows BEopt performing serial comparative analyses between corresponding EnergyPlus and TrueHome simulations.

Figure 2. Example mapping of walls in BEopt to EnergyPlus and TrueHome ....................................... 4

Figure 3. Workflow for simulations in BEopt using (a) EnergyPlus and (b) TrueHome ........................... 5

Figure 4. "Ideal" internal loads in the diagnostic building when evaluating HVAC categories .................. 8

Figure 5. Annual source energy consumption in EnergyPlus vs. TrueHome for a diagnostic building;

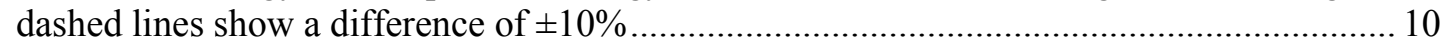

Figure 6. Annual source energy consumption in EnergyPlus vs. TrueHome for a typical building........... 11

Figure 7. Annual energy consumption for diagnostic buildings with varying refrigerator options (a) before and (b) after corrections

Figure 8. Annual energy consumption for diagnostic buildings with a slab foundation before corrections 13

Figure 9. Annual energy consumption for diagnostic buildings with a slab foundation after corrections.. 14

Figure 10. Annual energy consumption for diagnostic buildings with a miscellaneous electricity consumption before corrections

Figure 11. Annual energy consumption for diagnostic buildings with a miscellaneous electricity consumption after corrections

Figure 12. Annual energy consumption for diagnostic buildings with a boiler before corrections ............ 17

Figure 13. Annual energy consumption for diagnostic buildings with a boiler after corrections ............... 17

Figure 14. Annual results for water heating options in the diagnostic building ..................................... 19

Figure 15. Annual results for lighting options in the diagnostic building.......................................... 20

Figure 16. Annual results for unfinished attic options in the diagnostic building .................................. 21

\section{List of Tables}

Table 1. Characteristics of the Diagnostic and Typical Buildings ......................................................... 7

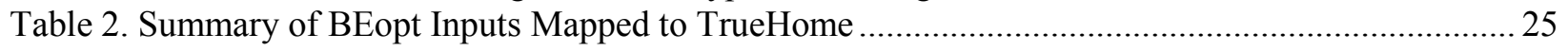

Table 3. BEopt TrueHome/EnergyPlus Test Suite Coverage ….............................................................. 29 


\section{Overview}

Building energy models can be used to estimate the energy consumption of buildings as well as the energy savings and cost-effectiveness of upgrades. However, it is essential that the building model provides accurate results so that there can be confidence in these predictions. There are several simulation engines that can be used to model residential buildings. This work details a comparison of two engines, EnergyPlus ${ }^{\mathrm{TM}}$ and Tendril TrueHome, to identify energy consumption discrepancies, understand the root cause and, if possible, rectify the discrepancy. This sort of software-to-software validation has potential to improve the ability of both engines to provide accurate results.

Tendril TrueHome is a physics-based simulation engine developed by Tendril to provide energy savings predictions for residential buildings to utility customers (Tendril 2014). EnergyPlus is the U.S. Department of Energy's (DOE's) flagship building simulation engine, designed to model both residential and commercial buildings (Department of Energy 2016). This study used the Building Energy Optimization Tool ${ }^{\mathrm{TM}}$ (BEopt), the National Renewable Energy Laboratory's (NREL's) residential energy system optimization program was used to automate comparisons of energy predictions between Tendril and Energy Plus.

While BEopt is typically used to perform simulations using a single engine (EnergyPlus), it can also be configured to work with multiple simulation engines. In this way, it is an ideal tool to compare results between simulation engines by providing identical inputs to each (Figure 1). BEopt also has a built-in test suite designed to facilitate engine-to-engine comparisons, which quickly identifies differences across a broad range of building configurations. This allows developers to quantify the impact of different algorithms and assumptions used in the engines as well as to quickly identify bugs. 

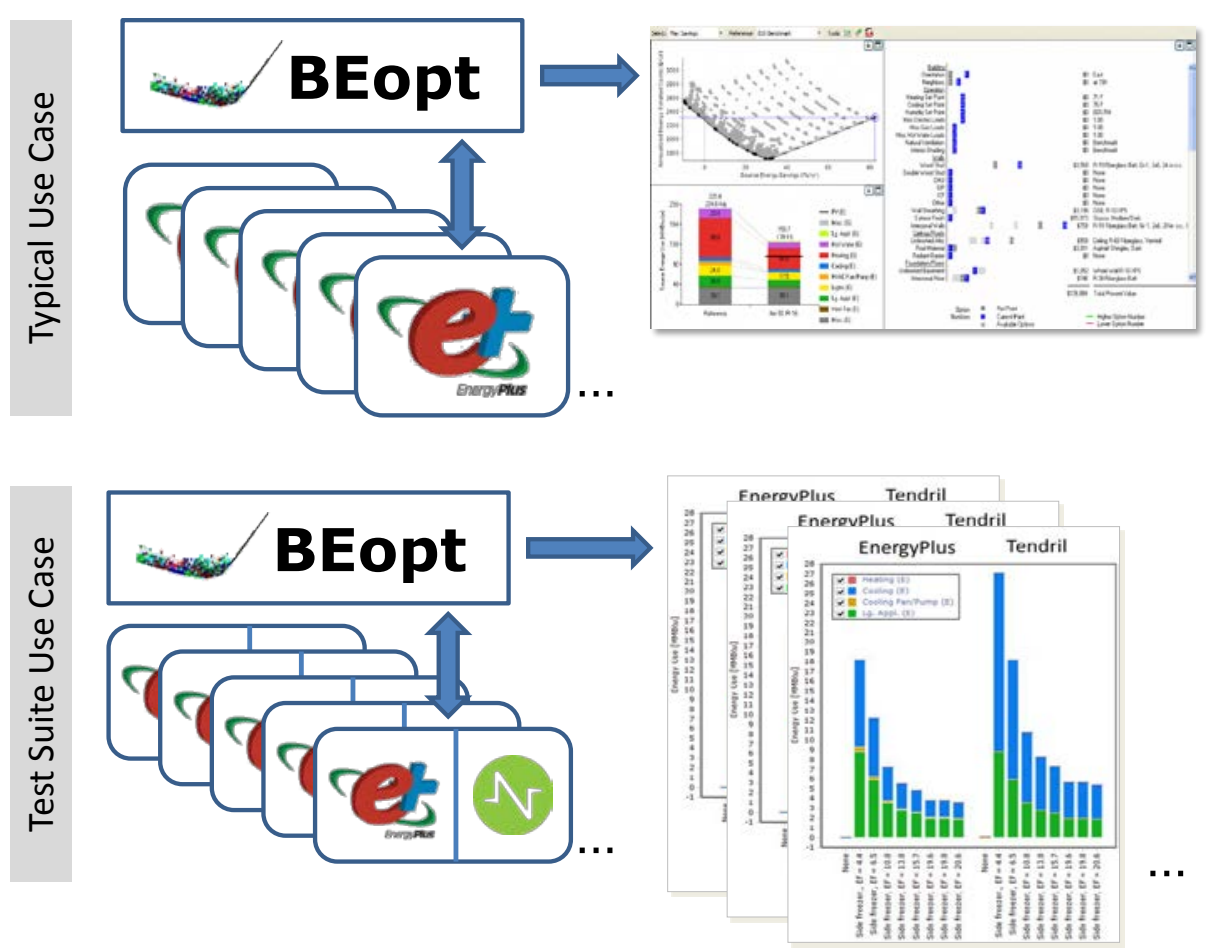

Figure 1. Schematic of BEopt use cases. Top shows BEopt performing optimization involving multiple EnergyPlus simulations. Bottom shows BEopt performing serial comparative analyses between corresponding EnergyPlus and TrueHome simulations. 


\section{Approach}

This study used an engine-to-engine comparison approach inspired by ASHRAE Standard 140 (Judkoff and Neymark 2006) to identify output differences between EnergyPlus and TrueHome. This approach has previously been used to compare other simulation engines to EnergyPlus (Horowitz et al. 2016), making it ideal for this project.

BEopt is a "meta" program that can work with multiple simulation engines. It provides a graphical user interface for quickly entering different building geometries, option characteristics (wall constructions, equipment specifications, etc.), and site characteristics. For comparison purposes, it also provides a test suite capability that runs through all of the different options in multiple simulation engines. This allows for a large number of simulations to be quickly performed with identical building configurations in both engines, which facilitates engine-toengine output comparisons.

When comparing energy simulation engines, output differences may occur for a variety of reasons. These include:

- Recommended inputs for technology models

- Available options

- Inputs for technology models

- Built-in assumptions and values

- Physics models and algorithm choices

- Coding errors in simulation engines.

In cases where there are differences due to recommended inputs used to model a certain technology, differences in algorithms, or differences in assumptions and values, it is not always practical to reconcile the differences. For each discrepancy shown here, the cause of the discrepancy is discussed along with the potential resolution, if available. Appendix A lists all technologies available in the current version of BEopt (2.7) and notes if they were included as part of this comparison. Appendix B lists the options in each category. Some technologies were excluded because they could not be modeled in TrueHome. In a few cases, a technology is excluded from the analysis even if it is available in both engines because of low market penetration in U.S. homes.

\subsection{Mapping}

The analysis starts by identifying equivalent building energy design options within each engine. Mapping is the process of creating equivalent inputs in both simulation engines for a given BEopt option. In BEopt, physical characteristics of a building are referred to as technologies or categories. Within each category, individual options represent a specific efficiency level, such as an R-13 wall in the stud wall category. Mapping ensures that all options across all categories are based on the same input values and thus consistent between the two engines. An example of mapping walls is shown in Figure 2. The actual wall consists of multiple materials in a complex construction. Its overall thermal performance is defined by the properties of each material as well as the stud spacing and dimensions. In EnergyPlus, walls are modeled as a series of layers, each 
with their own thermophysical properties (density, specific heat, and thermal conductivity) and thickness. This requires that the stud and cavity material properties be combined into a thermally equivalent material. In TrueHome, only the thermophysical properties and thickness of the overall wall assembly are used as inputs (i.e., representing the wall as a single equivalent material).

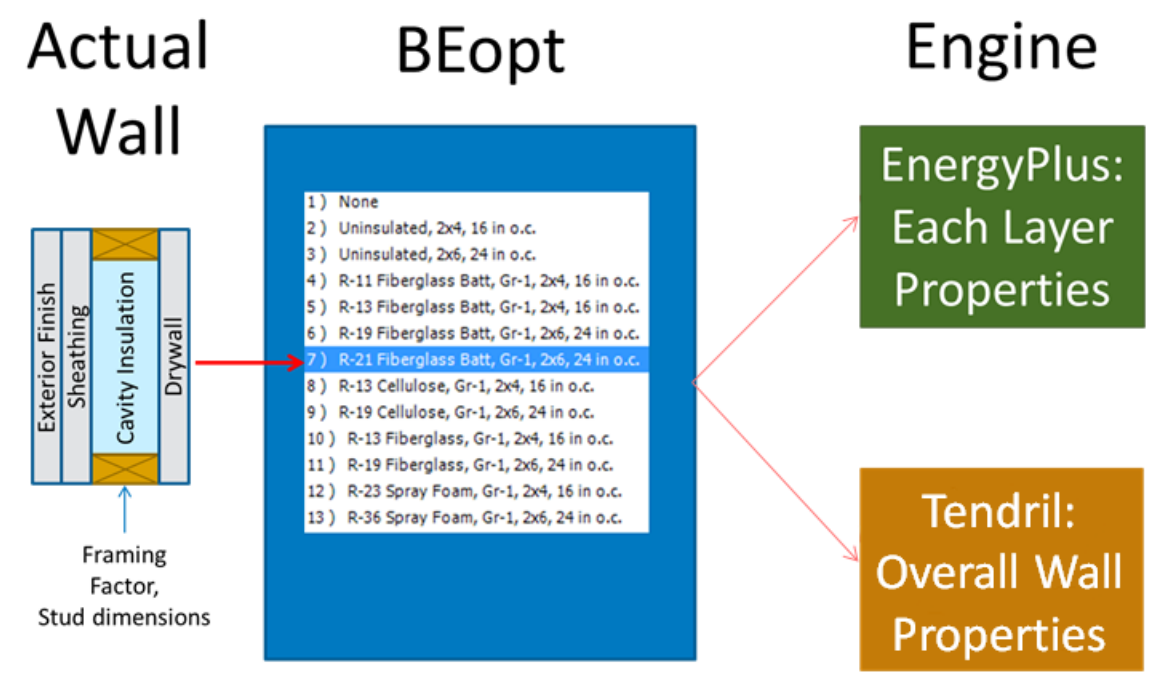

Figure 2. Example mapping of walls in BEopt to EnergyPlus and TrueHome

The complexity of mapping different building components for equivalent representation in multiple building simulation engines can vary significantly. In TrueHome, some components only require a few inputs (e.g., furnaces only require an overall efficiency). Others can be much more complex (e.g., anything that requires a custom schedule to be defined). Appendix A details some technology mapping solutions for both engines used by this study. In particular, Appendix A highlights cases where only certain options were mapped due to modeling limitations in one of the simulation engines - for example, constant year-round infiltration options as opposed to infiltration options that vary by wind speed and temperature.

\subsection{Workflow}

The basic workflow for running BEopt simulations in EnergyPlus is shown in Figure 3(a). The properties for all BEopt options across all available technologies are stored in a SQLite database $^{1}$. Each building model, characterized by its geometry and the technologies included in the home, is written to an .xml file. This .xml file is then processed by the EPlusInput.py python script, which creates a valid EnergyPlus input file. EnergyPlus is run using this input file with the appropriate weather data to create an output file, Outputs.csv. This file is then processed by the python script EPlusOutput.py, which creates an .xml file of the outputs. Finally, this is parsed by BEopt, which shows the results of the simulation.

Modifications to this process were made to allow TrueHome to be used with BEopt as shown in Figure 3(b) and include:

\footnotetext{
${ }^{1}$ https://www.sqlite.org/
} 
- Modifying the options library to only include options available in TrueHome

- Creating a Tendril.py python script to map BEopt inputs to TrueHome inputs

- Creating a TendrilOutput.py python script to map TrueHome outputs to BEopt outputs

- Modifying BEopt to automate the execution of the TrueHome process.

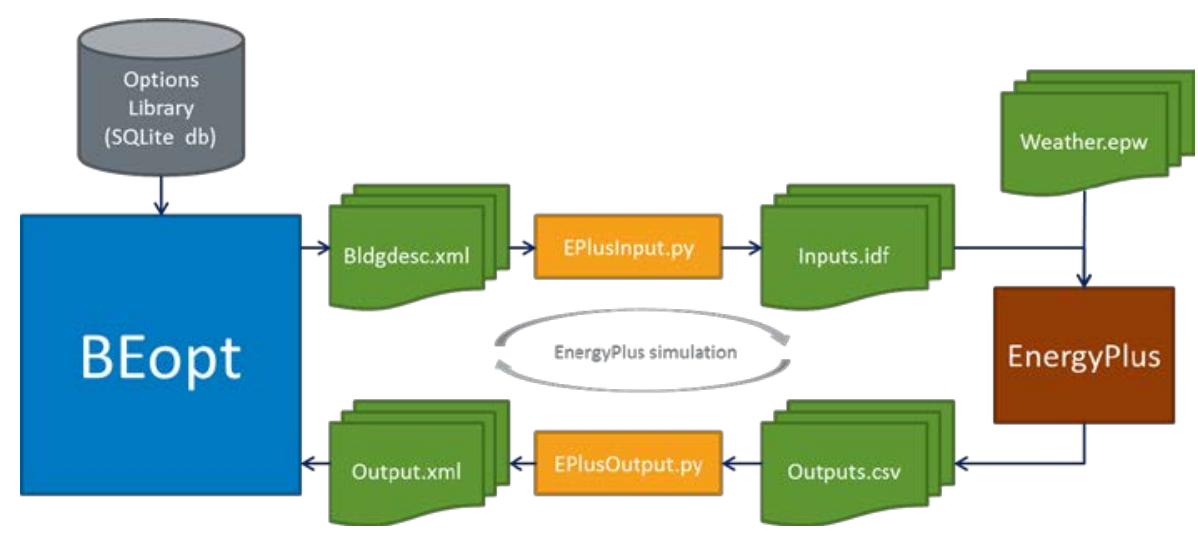

(a)

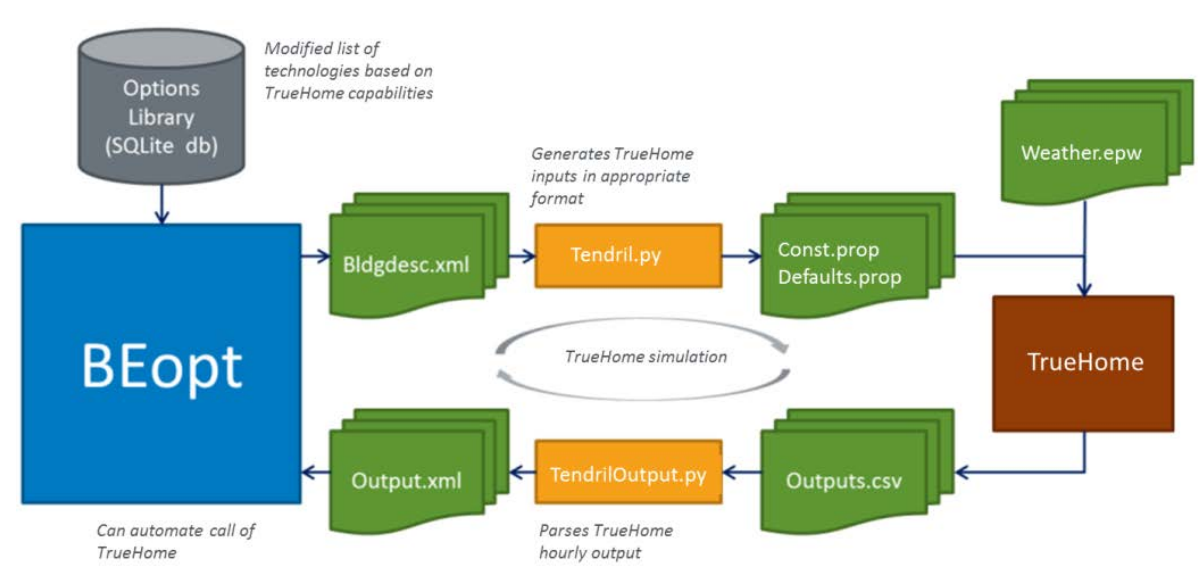

(b)

Figure 3. Workflow for simulations in BEopt using (a) EnergyPlus and (b) TrueHome

\subsection{Test Suite Setup}

The BEopt Test Suite is an automated tool that allows comparative simulations across multiple engines for tens of thousands of equivalent buildings. It systematically sweeps through each technology category in BEopt (for example, walls and water heaters), running each option one at a time. By doing this, it creates a sensitivity study over a large parameter space (Polly et al. 2012). The Test Suite was originally used for comparisons between the EnergyPlus and DOE-2 simulation engines (Booten et al. 2012). More recently, a similar project to this one connected BEopt to the Simple Energy and Enthalpy Model (SEEM) simulation engine used in the Pacific Northwest and performed test suite simulations to identify differences between the two engines (Horowitz et al. 2016). 
The test suite was modified to accommodate a range of buildings that can be modeled in both engines and contains:

- Two building types

○ Diagnostic building

○ Typical building.

- Six climates
○ Phoenix, AZ
- Houston, TX
- Atlanta, GA
- Los Angeles, CA
- Seattle, WA
○ Chicago, IL

- One geometry

- $1,200 \mathrm{ft}^{2}$, one story, slab, flat roof.

This results in 12 buildings in which every BEopt option was evaluated, one at a time, for a total of roughly 3,000 simulations in each engine. The diagnostic building is designed to isolate the impact of one particular technology on a building's energy consumption at a time by removing the impact of all other technologies. It includes superinsulated constructions; no internal gains; no windows; ideal heating, ventilation, and air conditioning (HVAC) equipment whenever possible; and zero infiltration. This was the primary building used for determining differences between the two engines since it allows options within each category to be compared independently from the other categories. Default options for the typical building are shown in Table $1 .^{2}$

This study included only a single geometry since TrueHome can only model flat roofs and slab foundations However, workarounds for unfinished attics were available that use flat roof constructions intended to approximate the impact of the unconditioned spaces without actually modeling additional zones. While some additional homes with unfinished attics were studied, runs in these homes were only performed for the attic categories rather than all available options. Results for these cases with attic workarounds are included in Section 3.3.3.

Of the six climates studied, this report presents results for Atlanta, Georgia, since that climate has both heating and cooling loads at significant levels.

\footnotetext{
${ }^{2}$ Note that while an "ideal" air conditioner, with a constant $100 \%$ efficiency, is available in both engines, it is not used here. The ideal air conditioner in EnergyPlus has a sensible heat ratio (SHR) of 0.8, while the ideal air conditioner in TrueHome has an SHR of 1. Increasing the SHR in EnergyPlus leads to psychrometric issues, while TrueHome doesn't track humidity or allow the efficiency to be set to any level other than 1 so a lower SHR cannot be simulated. Instead, an air conditioner with a seasonable energy efficiency ratio (SEER) of 13 is used in both engines.
} 
Table 1. Characteristics of the Diagnostic and Typical Buildings

\begin{tabular}{|c|c|c|}
\hline & Diagnostic & Typical \\
\hline Walls & Superinsulated & $R-13$ \\
\hline Finished Roof & Superinsulated & R-30 \\
\hline Slab & Superinsulated & Uninsulated \\
\hline Window Type & None & Double Pane, Low-e \\
\hline Window Area & None & $15 \%$ of Wall Area \\
\hline Infiltration & None & Constant $0.1 \mathrm{ACH}^{\mathrm{a}}$ \\
\hline Appliances & None & Standard \\
\hline Cooling Equipment & SEER $^{b} 13$ Air Conditioner & SEER $^{\mathrm{b}} 13$ Air Conditioner \\
\hline Heating Equipment & Electric Baseboard & $78 \%$ AFUE ${ }^{C}$ Furnace, Gas \\
\hline Water Heater & None & Gas $0.59 \mathrm{EF}^{\mathrm{d}}$ \\
\hline
\end{tabular}

When evaluating HVAC categories in the diagnostic building, "ideal" internal gains are added to the building. If these gains were not present, the heating and cooling loads in the building would be nearly zero since the envelope is superinsulated and there are no other gains in the building. The "ideal" internal gains are entirely sensible and have the same sinusoidal load shape for every day of the year. The magnitude of the gains varies seasonally, with the largest negative gains (which drive heating energy consumption) in the winter and the largest positive gains in the summer. The impact of these internal gains, and changes made to them over the course of this project, are discussed in Section 3.2.3. Figure 4 shows the annual internal gain used for the final results.

The typical building represents a new or recently constructed building, with construction practices and material characteristic found in new homes. While construction practices typically change with climate based on code requirements, only one set of default options can be set in the test suite. This home also has major appliances, lighting, and miscellaneous electric loads, which provide internal gains in the space. The options used for these homes represent typical electric appliances and the schedules are based on the Building America House Simulation Protocols (Wilson and Horowitz 2016). 


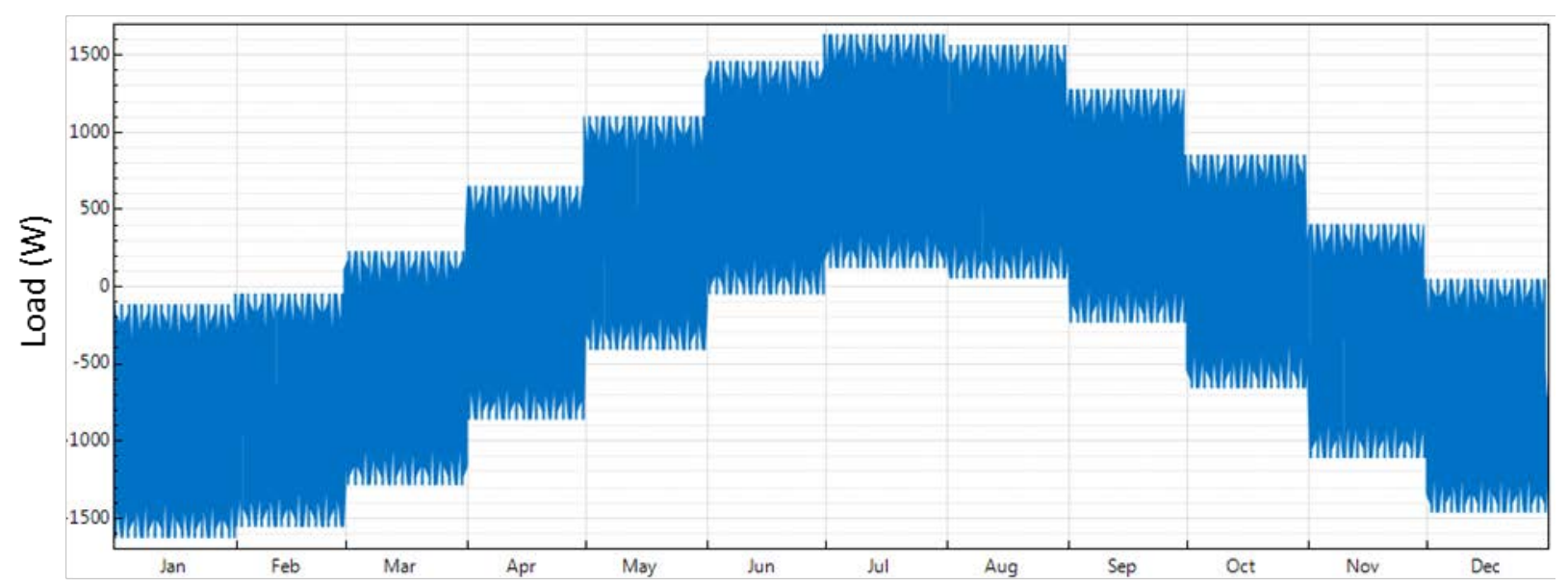

Figure 4. "Ideal" internal loads in the diagnostic building when evaluating HVAC categories

\subsection{Versions of EnergyPlus and Tendril TrueHome Used}

Due to the length of the project schedule, different versions of both EnergyPlus and Tendril TrueHome were used for the initial and final stages of this work. At the start of this work, EnergyPlus version 8.5 was used, while the final comparisons use EnergyPlus version 8.6. However, changes between EnergyPlus versions did not significantly affect any of the results for this comparison. The study also began with TrueHome version 3.8.4, and the corrections implemented, along with concurrent development by Tendril, led to version 3.9.11 by the end of this study. Unlike EnergyPlus, these TrueHome changes did impact the results of the comparison between engines. Relevant changes include:

- Fixes as described in subsequent sections.

- Changes in inputs for HVAC options. These changes set the HVAC efficiency to be a function of the age of the equipment rather than allowing the efficiency to be set directly. This prevents TrueHome from simulating some of the high efficiency HVAC equipment available in BEopt.

- Changes to the sensible heat ratio (SHR) and schedule of lighting and appliances. Some appliances and lighting had built in schedules and SHRs at the end of the project that could no longer be set through inputs. 


\section{Results}

The overall results presented here span all categories, with more detail given for categories that have unresolved discrepancies or cases in which a major fix was identified. In all categories, there are expected to be some minor output differences due to the intrinsic differences in algorithms and assumptions used in the engines. In many categories these differences are negligible. Cases where discrepancies in the results are significant are highlighted in this section along with some discussion of the root cause of large differences. When categories are investigated in more detail, results from the diagnostic building are shown to remove the impact of any interacting factors.

While many changes were made as a part of this project to produce more comparable results between Tendril TrueHome and EnergyPlus, not all of them are discussed here. In some cases, these were initial issues with the input mapping due to either coding errors or incorrect interpretations of the models in an engine. This section instead focuses on fundamental changes to the engines when modeling errors or bugs were discovered as well as of discrepancy between the two engines that were not fully resolved. As such, it highlights the major findings of this work and potential areas for further investigation.

\subsection{Overall Energy Consumption Comparison}

Final results (after discrepancies were resolved where possible) for annual energy consumptions in each engine are presented in two different ways. Figure 5 plots the annual source energy consumption in TrueHome and EnergyPlus against each other for the diagnostic building, with dashed lines showing differences of $\pm 10 \%$. In both cases, the building was simulated in Atlanta, Georgia, since Atlanta is a mixed climate has meaningful heating and cooling loads. The average difference between the two engines is $13 \%$, but for some cases there are significant discrepancies between the two. The most significant remaining discrepancies are for technologies with substantial differences in how they are modeled in the respective engines, which is explained in further detail in the subsequent sections. 


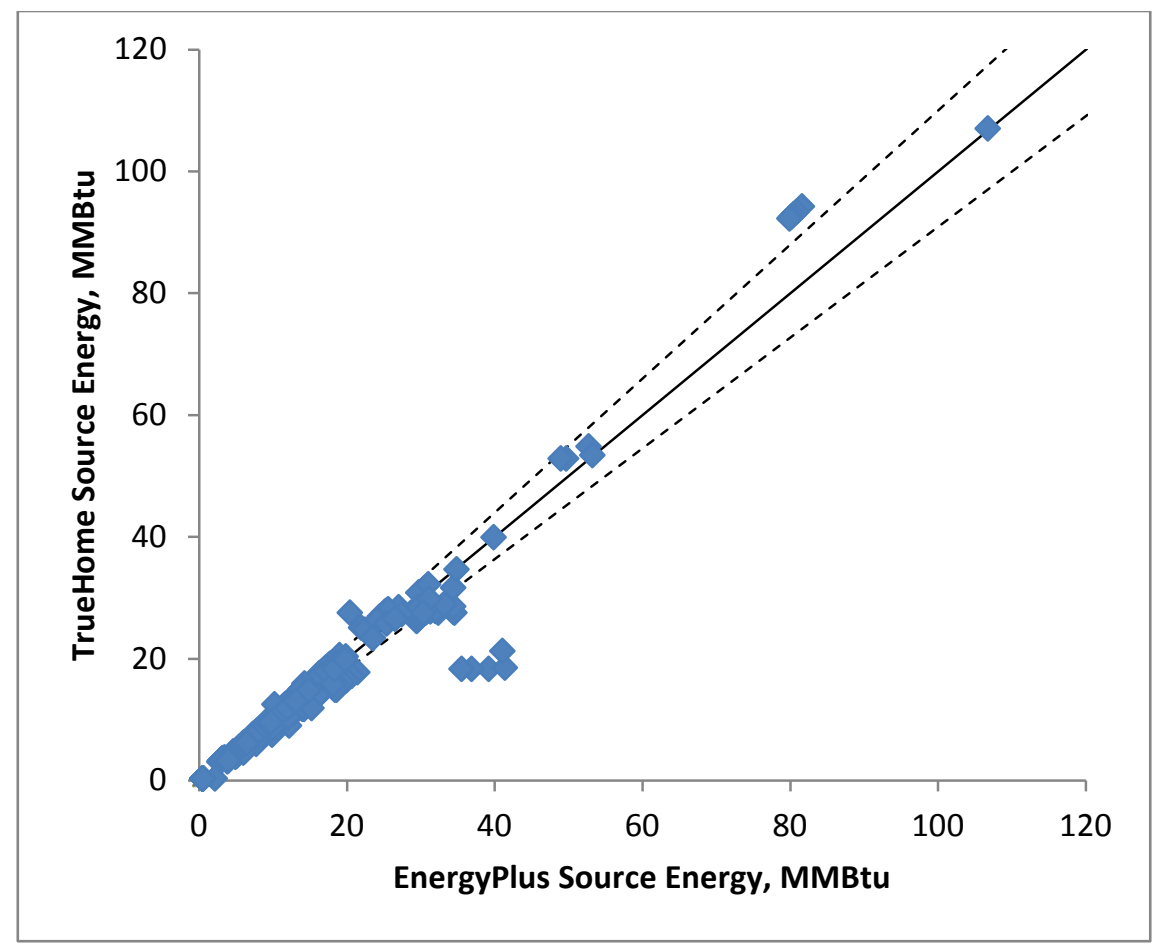

Figure 5. Annual source energy consumption in EnergyPlus vs. TrueHome for a diagnostic building; dashed lines show a difference of $\pm 10 \%$

Figure 6 shows the energy consumption buildings for a typical building in Atlanta, Georgia across all categories. While the typical building higher energy consumption than the minimal building, the discrepancies in this case are within 10\% for all homes, demonstrating good agreement between the two engines for the range of options tested here. In addition, there are no major outliers in the new construction building. The outliers that exist in the diagnostic building occur due to combinations of options - such as a heat pump water heater (HPWH) in a superinsulated building - that don't occur in typical homes. There is a slight bias in the results that EnergyPlus tends to predict lower annual energy consumption than Tendril, but it is small enough that it could easily be explained by the intrinsic differences in the models used by each engine. 


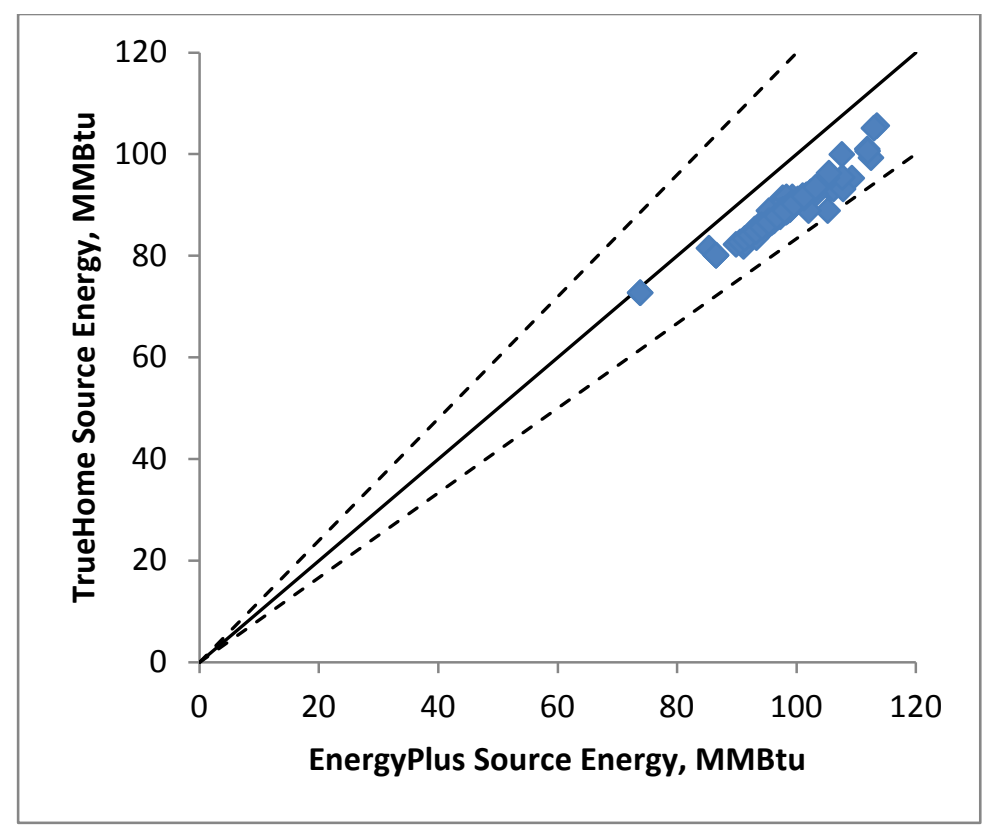

Figure 6. Annual source energy consumption in EnergyPlus vs. TrueHome for a typical building

\subsection{Discrepancies Corrected}

\subsubsection{Appliances}

A discrepancy was identified with appliances, as demonstrated by results for a diagnostic building with refrigerators. However, it was determined that this issue also occurs for any source of internal gains, including all appliances, lighting, occupants, and miscellaneous electric loads. Figure 7 (a) shows the initial annual energy consumption in both engines for varying refrigerator options, which highlights the issue. When no appliances are present in the home, there is no energy consumption as expected in the diagnostic building. When a refrigerator is added, its electrical energy consumption provides a sensible internal gain to the home. To offset this gain, an equal amount of cooling is required to keep the home at the setpoint temperature.

As expected, in EnergyPlus the cooling energy (with the diagnostic building's air conditioner) exactly offsets the refrigerator electrical energy consumption plus the cooling fan energy (which also becomes an internal gain). However, in TrueHome the amount of cooling is significantly higher than the heat added to the space from the refrigerator, indicating that the energy balance for the building is not being preserved. The cause of this issue turned out to be a bug related to how the HVAC energy consumption was calculated for the building. After correcting this issue, the results from TrueHome looked identical to EnergyPlus as shown in Figure 7 (b). 


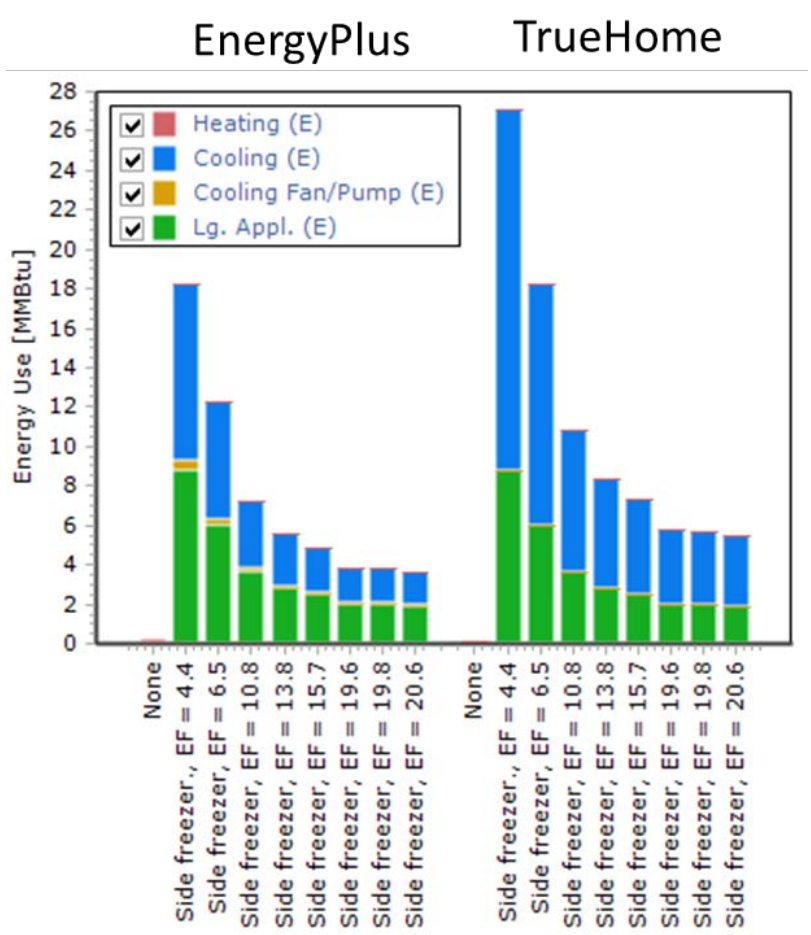

(a)

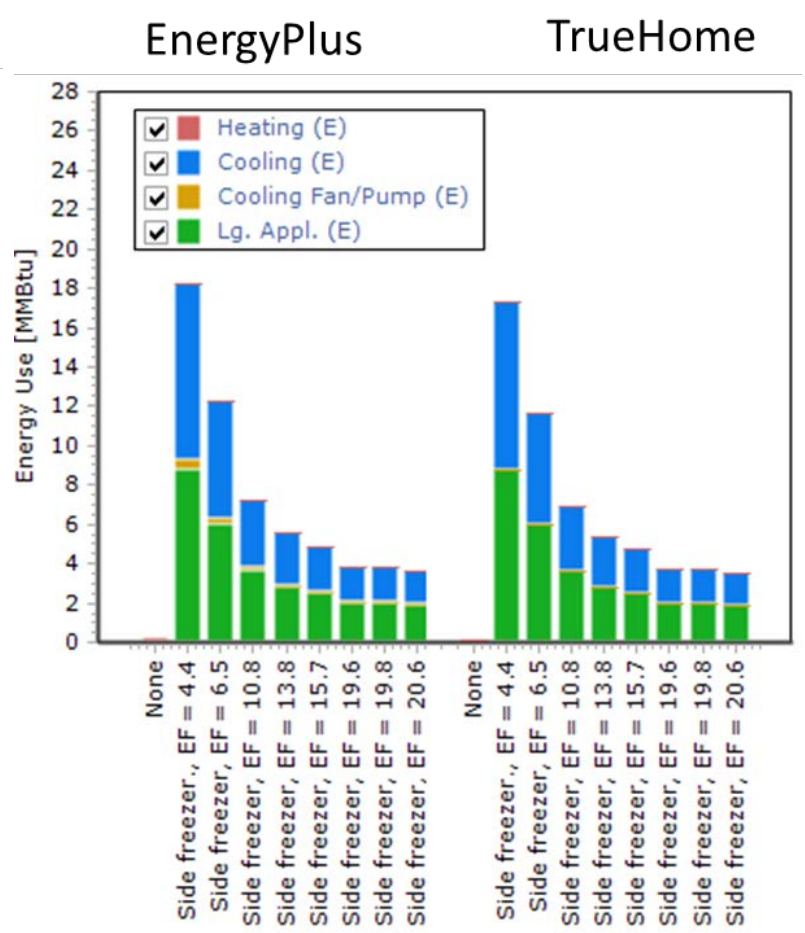

(b)

Figure 7. Annual energy consumption for diagnostic buildings with varying refrigerator options (a) before and (b) after corrections

\subsubsection{Slab Foundations}

Since TrueHome is a single-zone model, the only common foundation type it can handle without a workaround is a slab (TrueHome can also model pier and beam foundations, although they are uncommon in the United States and were not compared here). To model slab foundations in TrueHome, the initial approach included the thermal properties of the slab and any carpet/insulation as a single construction. This construction would then be modeled as any other construction layer except with the ground temperature as the outside boundary condition and no impact from wind or solar radiation. One downside to this approach is that it cannot be used to model situations where insulation is only applied to certain portions of the slab (e.g., perimeter insulation). As a result, the initial comparison only evaluated whole-slab insulation levels.

BEopt implements a foundation model in EnergyPlus based on the Winkelmann method (Winkelmann 2002). This method specifies a construction with layers representing the slab (including any floor finishes), one foot of soil, and a fictitious insulation layer. The properties of this insulating layer are derived from analysis using a two-dimensional finite-difference approach (Huang et al. 1988) and vary depending on the insulation configuration and R-value to achieve the appropriate foundation heat transfer. Initial results showing the comparison between the two engines are shown in Figure 8. 


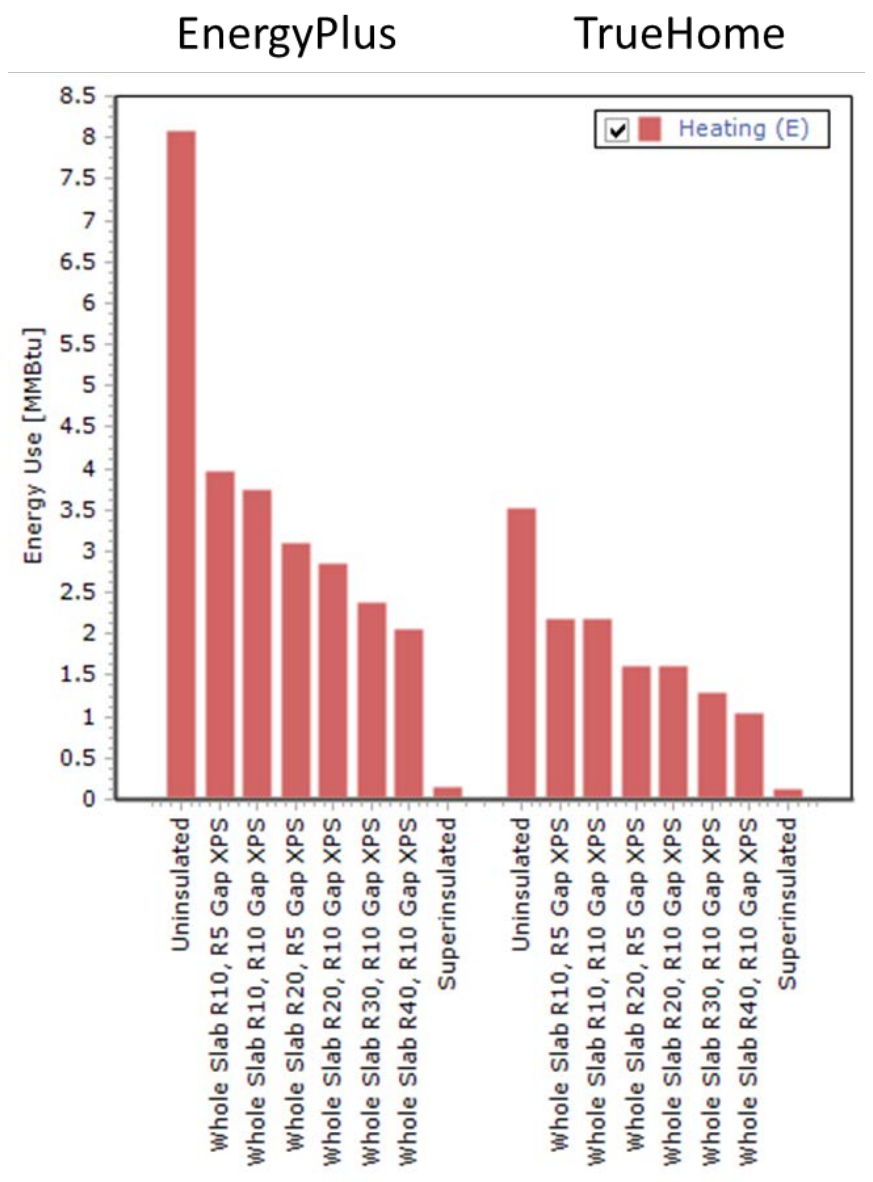

Figure 8. Annual energy consumption for diagnostic buildings with a slab foundation before corrections

Beyond not accounting for many of the possible configurations of slab insulation found in residential buildings, TrueHome also showed much less foundation heat transfer than the EnergyPlus model. Based on these comparisons, NREL recommended and Tendril implemented the Winkelmann approach in TrueHome. After implementing this approach, nearly identical results were obtained as shown in Figure 9. 


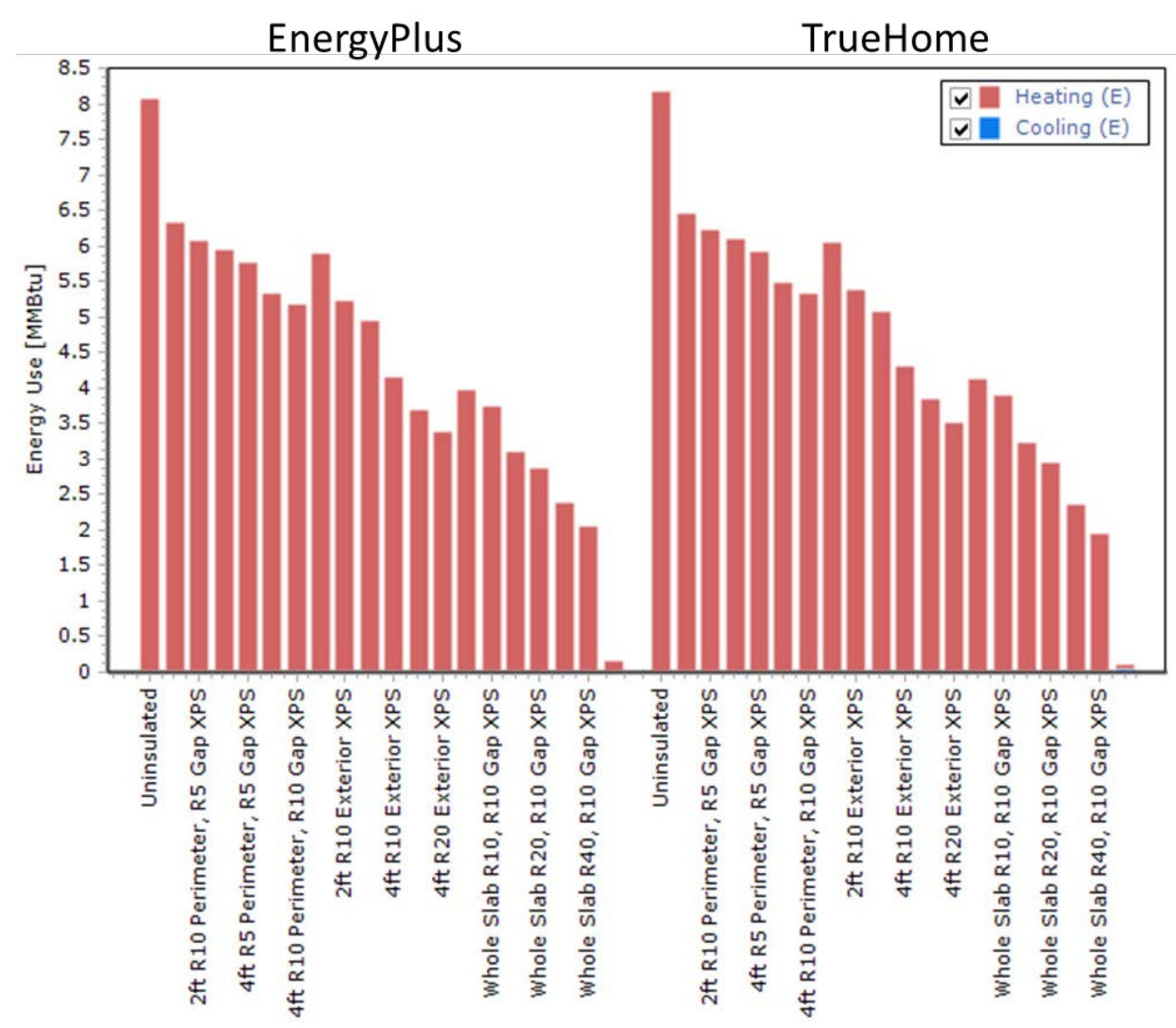

Figure 9. Annual energy consumption for diagnostic buildings with a slab foundation after corrections

\subsubsection{Ideal Gains}

As previously mentioned, an "ideal" internal gain is specified in the diagnostic building when evaluating HVAC options to ensure that a large, known quantity of both heating and cooling are required throughout the year. This internal gain initially had a magnitude of about 7,500 watts (W). Initial results, shown in Figure 10, found substantially lower heating and cooling energy consumption in TrueHome compared with EnergyPlus. 


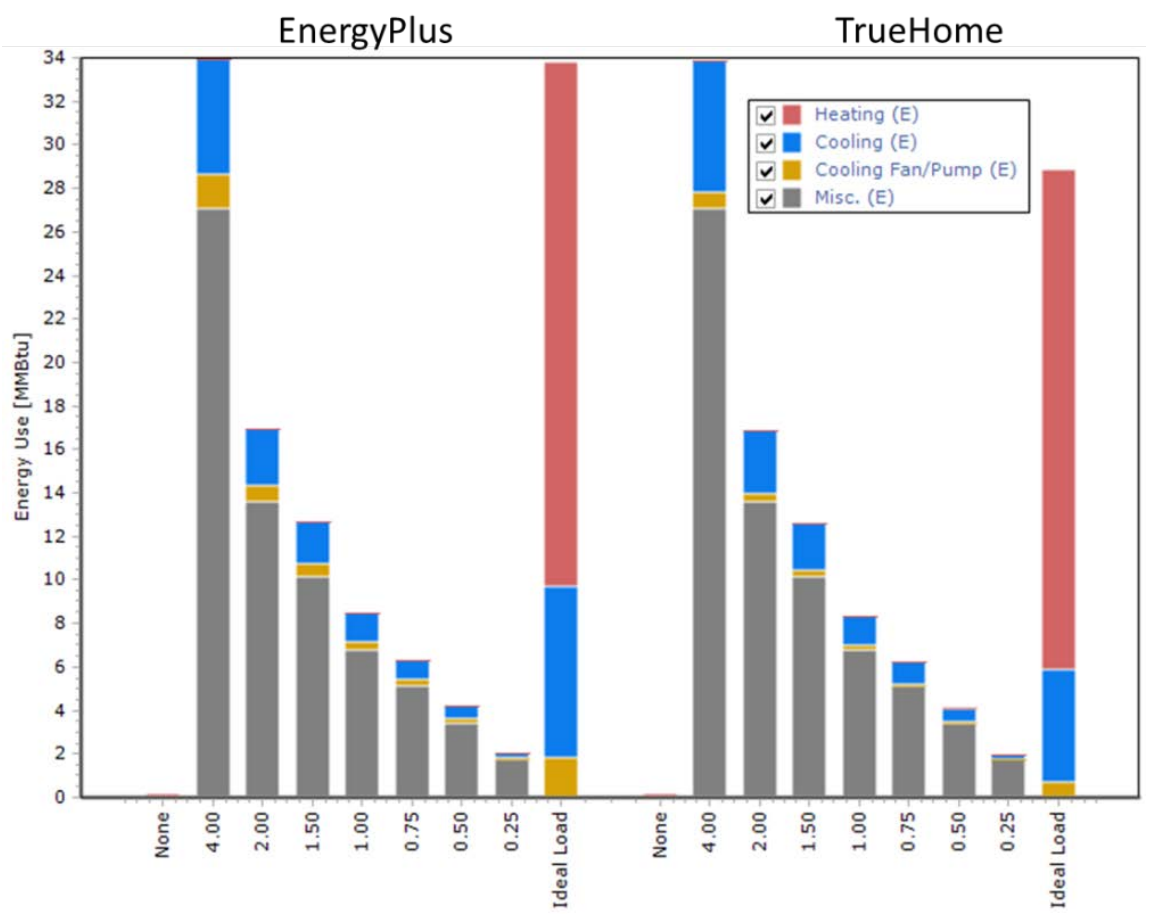

Figure 10. Annual energy consumption for diagnostic buildings with a miscellaneous electricity consumption before corrections

After investigating this discrepancy, it was determined that TrueHome model convergence issues arose from internal gains of this magnitude that rapidly changed. While TrueHome had no issue converging with smaller gains or large gains with a more typical usage schedule, large gains that rapidly change over time such as the "ideal" gains led to cases where the calculations of the heat loss from the building did not converge, leading to erroneous HVAC energy consumption predictions. As a result, the magnitude of this gain was changed to about 1,500 W, which is still large enough to give substantial heating and cooling loads without causing any convergence issues. The issue where rapidly changing large gains may cause issues has been reported to Tendril. Results after making this change are shown in Figure 11. 


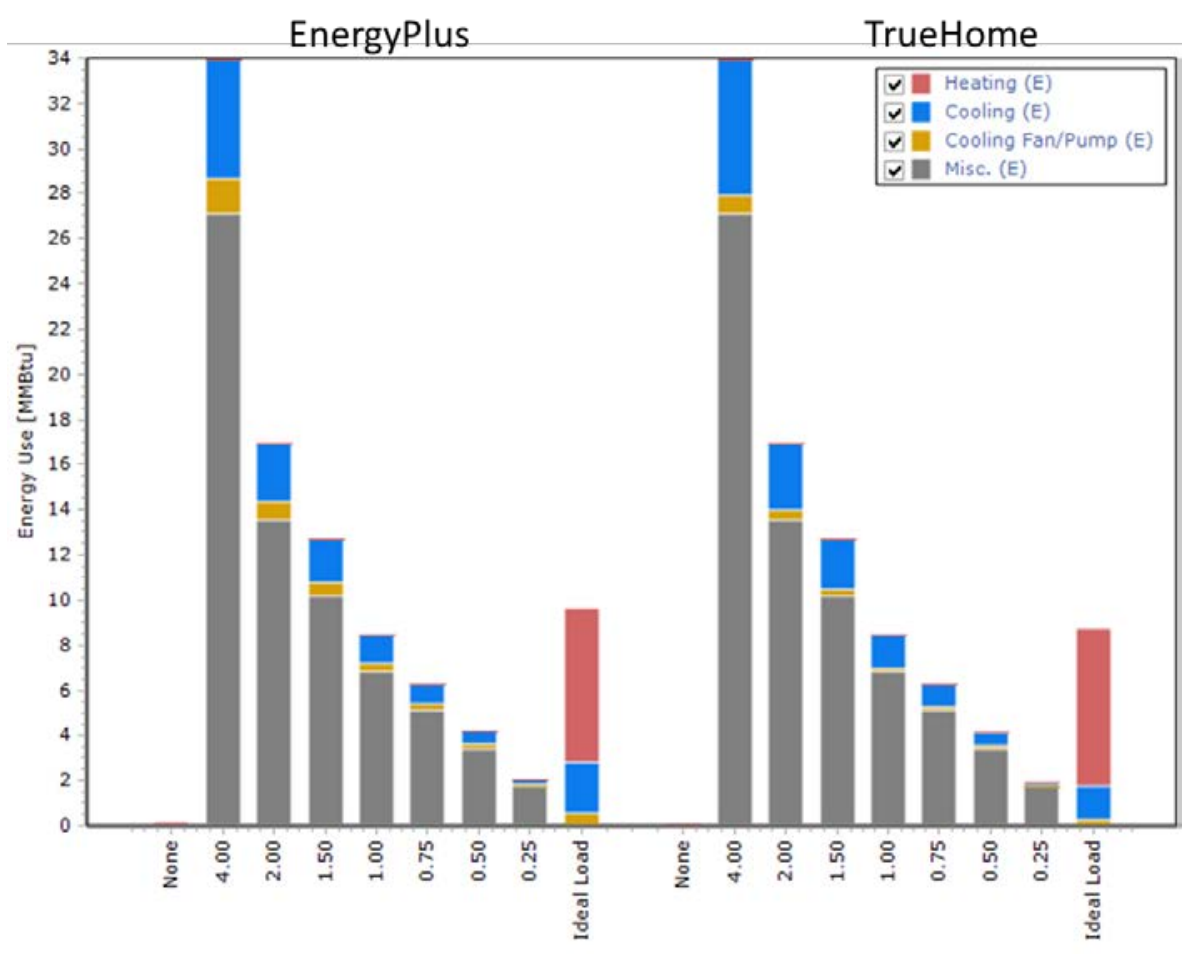

Figure 11. Annual energy consumption for diagnostic buildings with a miscellaneous electricity consumption after corrections

\subsubsection{Boilers}

Figure 12 shows the results for different boiler options in EnergyPlus and TrueHome in the diagnostic building. The most notable trend is that heating energy consumption for boilers is consistently higher in TrueHome (the difference in cooling energy consumption is due to intrinsic air conditioner model differences when using the ideal loads and shows up in every HVAC category). When modeling boilers in EnergyPlus, the pump is set up to run at full power at any time there is a call for heating, even if the boiler is modulating. However, a workaround is used when reporting the boiler pump energy consumption that takes into account this modulation. Since the boiler pump energy consumption becomes useful heat to the water used for space heating, this leads to the overall heating-plus-pump energy reported by EnergyPlus to be less than what is actually used for heating. This implementation in EnergyPlus has since been fixed, leading to the better agreement shown in Figure 13. 


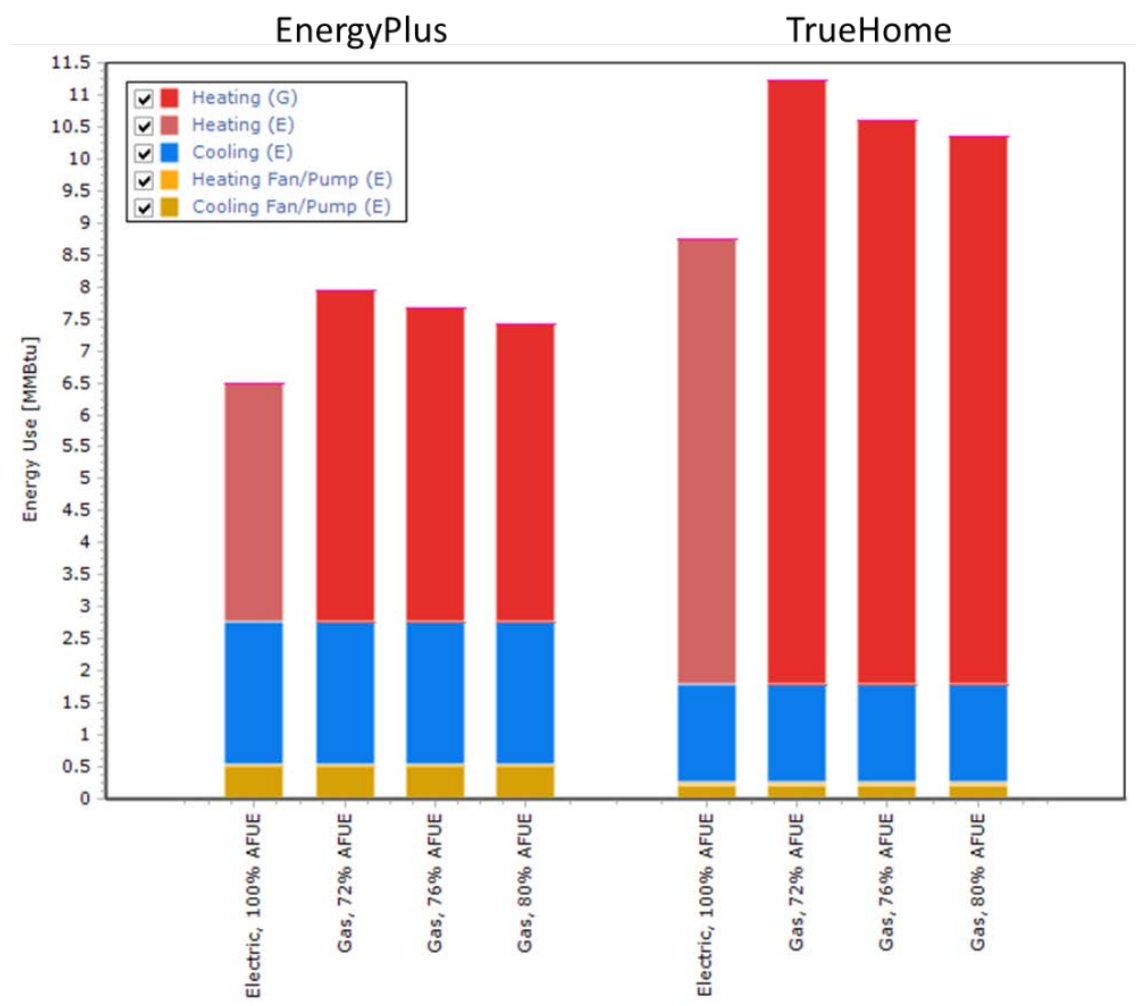

Figure 12. Annual energy consumption for diagnostic buildings with a boiler before corrections

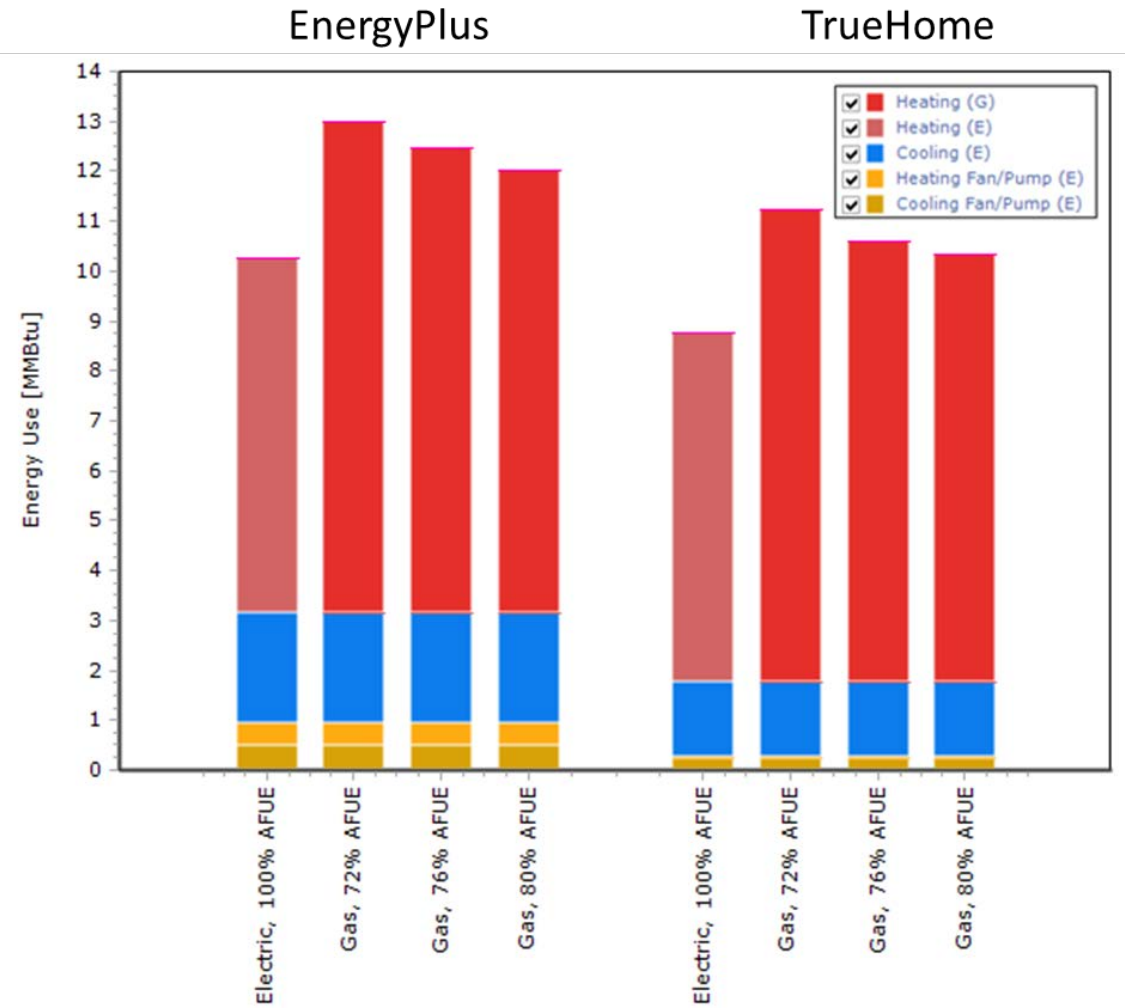

Figure 13. Annual energy consumption for diagnostic buildings with a boiler after corrections 


\subsection{Discrepancies Partially Investigated}

\subsubsection{Water Heating}

Figure 14 shows the annual results in the diagnostic building for all options in the water heating category. By far the largest differences are in cases where a HPWH is evaluated. Note that EnergyPlus splits HPWH energy consumption to main and supplemental energy use depending on whether the heat pump or electric elements are used to reheat the tank. In TrueHome, the HPWH is defined only by the COP of the water heater, with the load always being met by a heating element with that COP. The EnergyPlus HPWH model is considerably more sophisticated and has been validated against both laboratory data and other models (Horowitz et al. 2016). It includes a heat pump with a COP that varies as a function of ambient air wet bulb temperature and the temperature of water in the tank. The EnergyPlus HPWH model also includes backup electric resistance elements; control logic, based on laboratory measured HPWH performance, determines whether the heat pump or electric resistance elements are used to meet the load. In addition, the EnergyPlus heat pump pulls heat out of the ambient air around it, which leads to the HPWH interacting with the building HVAC system, whereas HPWHs in TrueHome have no impact on the building loads.

There are several reasons for the discrepancies that occur across all options. Both engines use the same algorithm for the mains water temperature, but use different draw profiles. EnergyPlus is run using an annual schedule of discrete hot water events for each water heating end use, while TrueHome uses smooth hourly profiles for the hot water use. These draw profiles have the same annual draw volume, but the EnergyPlus discrete events may lead to some cases where the water heater is unable to meet the load due to the potential for events in the annual schedule to stack, which may remove heat from the tank faster than the heat sources can add it. However, these instances of stacking are infrequent and would only lead to a slight annual discrepancy between the two engines. 


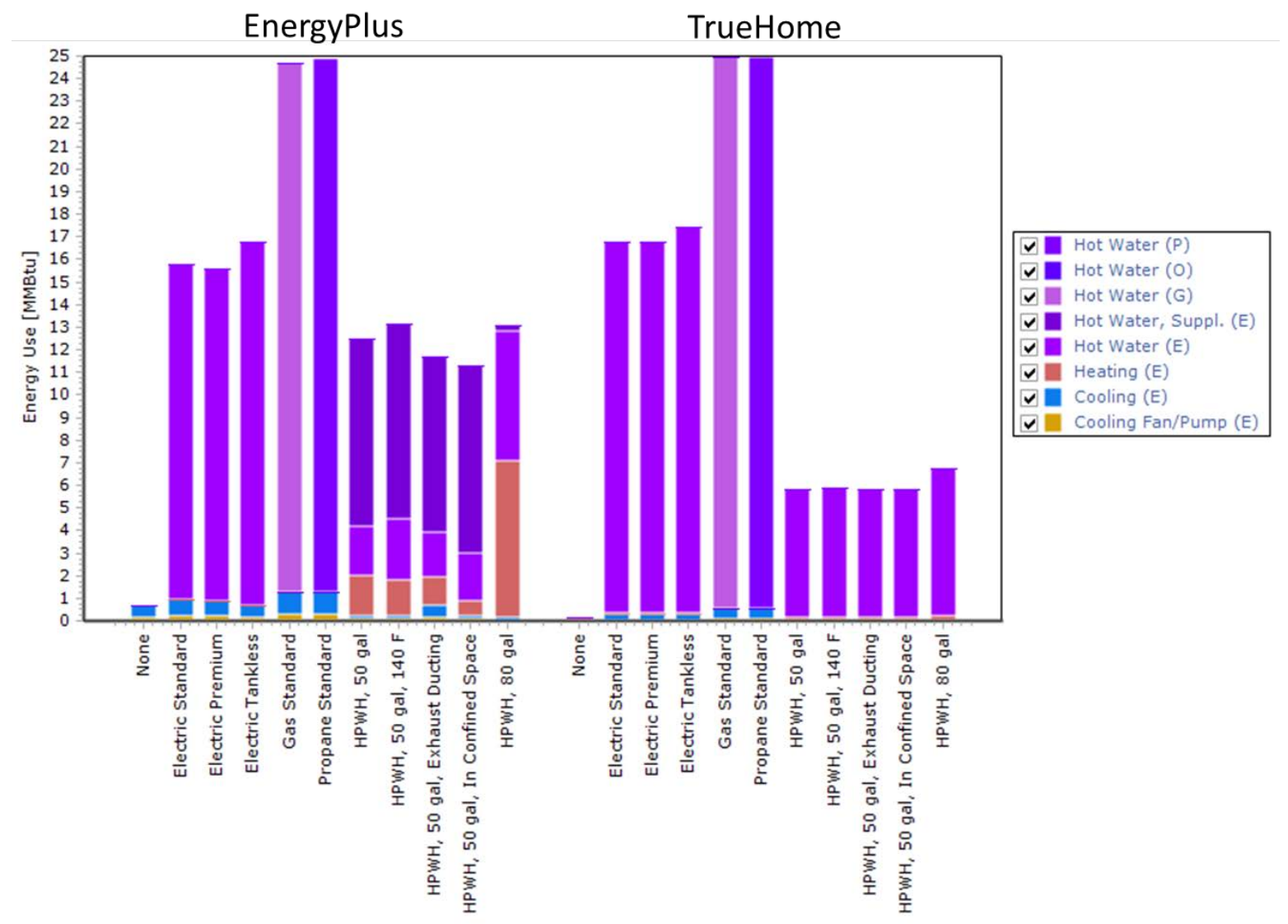

Figure 14. Annual results for water heating options in the diagnostic building

\subsubsection{Lighting}

Figure 15 shows the results for various lighting options in the diagnostic building. Across all options, EnergyPlus has higher direct lighting energy consumption than TrueHome. Since the lighting energy consumption becomes an internal gain, the engines' outputs also reflect differences in heating and cooling end uses. However, $100 \%$ of the lighting energy consumption in EnergyPlus becomes an internal gain, while in TrueHome this fraction is $90 \%$. Some of the heat emitted by the lights may end up outside of the space where the light is located (for example transmitted out the window or conducted into an adjacent zone in the case of recessed lighting), so this difference amounts to a difference in assumptions about where the heat generated ultimately ends up. 


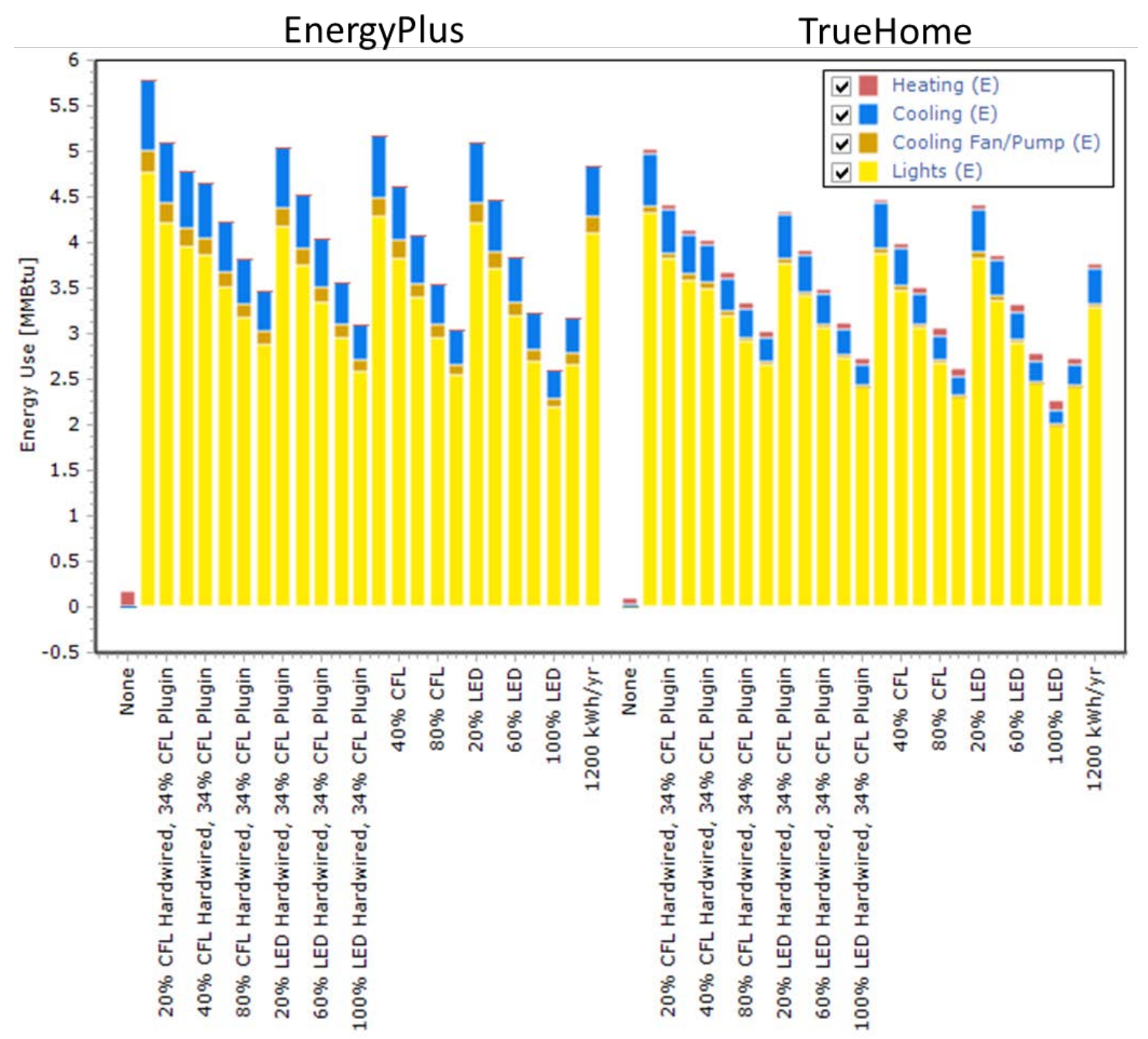

Figure 15. Annual results for lighting options in the diagnostic building

While the same lighting power level is specified in both engines, there are different schedules used. In EnergyPlus, the schedule is based on the Building America House Simulation Protocols (Wilson and Horowitz 2016). This schedule, which changes based on the location's latitude/longitude, changes both the daily profile and the magnitude of lighting energy consumption seasonally to account for the change in daylight hours over the year. The TrueHome schedule also has seasonal variations, but differences in the algorithms lead to slightly different annual on times for lighting. These differences in schedules contribute to the overall discrepancies observed in lighting energy consumption.

\subsubsection{Unfinished Attics}

Figure 16 shows the annual energy consumption for diagnostic buildings with unfinished attics in EnergyPlus and TrueHome. TrueHome doesn't have the capability to explicitly model attics, as it is a single zone model that only supports flat roofs. However, Tendril has developed a series of finished roof constructions representing different R-values that were designed to capture the impact an unfinished attic has on the overall building energy consumption. Results shown here are for cases using this workaround. 


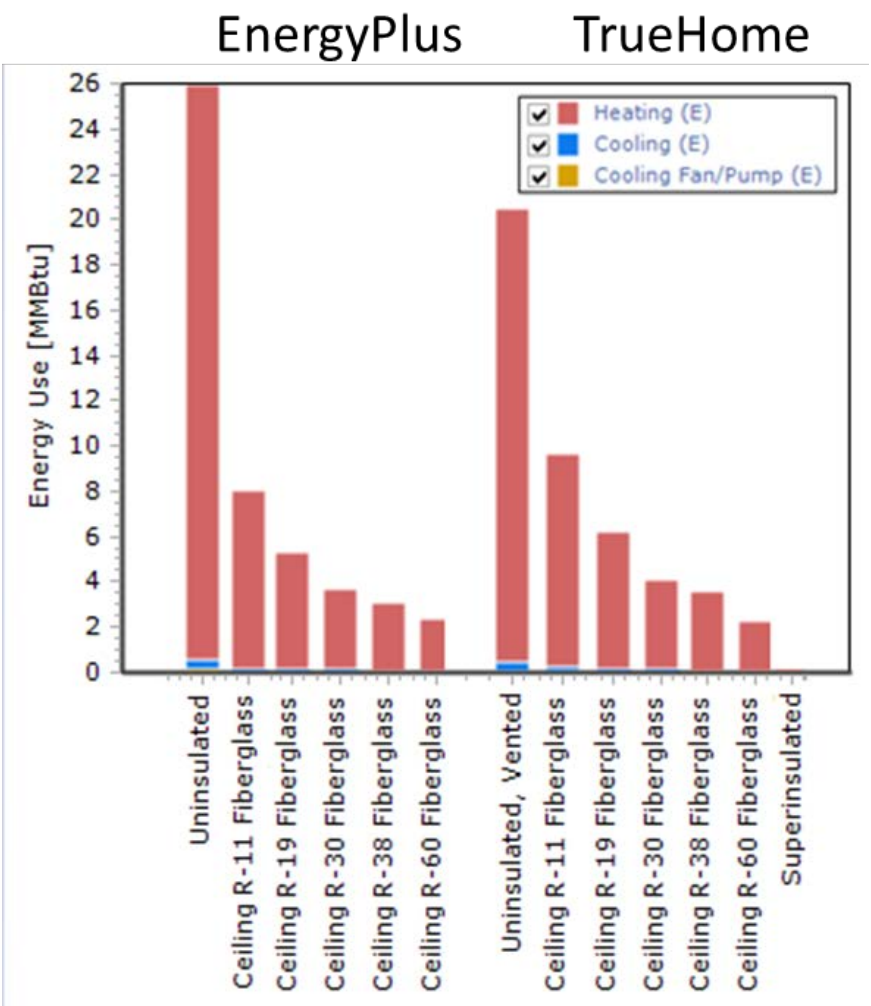

Figure 16. Annual results for unfinished attic options in the diagnostic building

When using the workaround for attics, the EnergyPlus model shows higher energy consumption for uninsulated cases and lower energy consumption for cases with insulation. The largest discrepancy is for an uninsulated attic and the magnitude of the difference decreases as the attic $\mathrm{R}$-value increases. This trend held across all climates studied as is expected as differences between the models become less important as the overall amount of heat transferred through the attic becomes small relative to the other surfaces. The discrepancy is almost $20 \%$ for cases with uninsulated attics. For attic $\mathrm{R}$ values greater than R-11, the discrepancy decreases to less than $10 \%$. TrueHome's workarounds for the attics has several other limitations. The results shown here are for cases of a 1,200 $\mathrm{ft}^{2}$, one story home with a $6: 12$ pitch gable roof. The roof pitch was chosen based on the default value used by BEopt and is assumed to be representative of a typical home. However, this workaround may not suffice for cases with different roof pitches or for other roof types (for example, hip roofs). In addition, since the attic is not explicitly modeled, no equipment can be modeled in the attic. This limitation is most significant for duct systems, which are located in the attics of many homes in hot climates. However, since TrueHome can't model ducts, this impact cannot be quantified here.

\subsection{Categories Excluded}

Not all of the technologies that are available in BEopt can be modeled in TrueHome. In particular, TrueHome does not currently include the capability to model ducts, below-grade or unconditioned zones (although workarounds were used for cases with unfinished attics as previously described), indoor moisture, or mechanical ventilation. As a result of these limitations, not all categories that are available in BEopt were mapped for TrueHome, which led to these cases not being explored as part of this project. Appendix A includes the full list of 
technologies indicating which were mapped, partially mapped or excluded. Of the technologies not included in this work, several are common in single family homes. For example, ducts cannot currently be modeled in TrueHome. For homes with forced air HVAC equipment, this limitation essentially means that TrueHome is modeling ducts located in conditioned space. Explicitly modeling ducts would also require that unconditioned zones (where the ducts could be located) be included in the model to correctly account for duct losses/gains (through conduction and/or leakage) into those zones. However, an alternate approach would be to calculate the seasonal distribution system efficiency (DSE) for the ducts and build the impact of DSE into TrueHome. An approach to determine the DSE for different duct locations, ducting system configurations, HVAC equipment, and climates has been developed as part of ASHRAE standard 152 (ASHRAE 2014). This approach could potentially be incorporated into TrueHome without requiring additional zones to be simulated, leading to only a modest increase in runtime while still capturing the impact from ducts in a home. Tendril has been made aware of the DSE approach and is currently evaluating including it in future versions of TrueHome.

Since TrueHome is a single zone model, below-grade and unconditioned zones cannot be explicitly modeled. Many single family homes have attics, garages, basements, and/or crawlspaces. For homes with an unfinished attic, the workaround employed by TrueHome showed good agreement for insulated attics in the cases explored here. However, workarounds for other unconditioned zones were not explored. Of the unconditioned zones typically found in homes, unfinished basements and crawlspaces have the largest impact on building loads and remain unexplored. The complexities of foundation heat transfer make these more challenging to model through a workaround than unfinished attics. TrueHome provides some constructions to represent homes with these foundation types, but they were not studied during this project due to time and budget restrictions. 


\section{Conclusions}

Comparative output analysis was performed through BEopt to determine the differences in energy consumption predictions between the EnergyPlus and the Tendril TrueHome engines. The BEopt Test Suite allowed thousands of simulations to be automatically compared between the two engines across a range of building characteristics and climates. As a result of this work, several discrepancies and opportunities for improving input assumptions were identified and adjustments made to TrueHome. Building features that occur in a large number of single family homes that cannot be modeled in TrueHome were also identified along with potential workarounds or suggested modeling approaches. In cases where discrepancies still exist, the cause of this discrepancy has been proposed and potential resolutions have been provided as appropriate to the developers of both tools. The final results show that the differences in annual energy between typical buildings representative of new construction homes are within 10\% for the cases considered here. 


\section{References}

ASHRAE. (2014). Standard 152-2014: Method of Test for Determining the Design and Seasonal Efficiencies of Residential Thermal Distribution Systems. Atlanta, GA: ASHRAE/ANSI.

Booten, C., Kruis, N., and Christensen, C. (2012). Identifying and Resovling Issues in EnergyPlus and DOE-2 Window Heat Transfer Calculations. Golden, CO: National Renewable Energy Laboratory.

Department of Energy. (2016). EnergyPlus Documentation. Washington, DC: Department of Energy.

Horowitz, S., Maguire, J., Tabares, P., Winkler, J., \& Christensen, C. (2016). EnergyPlus and SEEM Modeling Enhancements via Softwater-to-Software Comparisons Using NREL's BEopt Test Suite. Golden, CO: NREL.

Huang, Y., Shen, L., Bull, J., and Goldberg, L. (1988). Whole-House Simulation of Foundation Heat Flows Using the DOE-2.1C Program. ASHRAE Transactions 94(2).

Judkoff, R., and Neymark, J. (2006). Model Validation and Testing: The Methodological Foundation of ASHRAE Standard 140. ASHRAE 2006 Annual Meeting (pp. 24-29). Quebec City, Canada: ASHRAE.

Polly, B., Horowitz, S., Booten, C., Kruis, N., and Christensen, C. (2012). Automated Comparison of Building Energy Simulation Engines. Golden, CO: NREL.

Tendril. (2014). The Tendril Physics-based Building Model. Boulder, CO: Tendril.

US Department of Energy. (2016). EnergyPlus Documentation. Washington, DC: US Department of Energy.

Wilson, E., \& Horowitz, S. (2016). 2016 Building America Housing Simulation Protocols. Golden, CO: NREL.

Winkelmann, F. (2002). Underground Surfaces: How to Get a Better Underground Surface Heat Transfer Calculation in DOE-2.1E. Building Energy Simulation User News, 5-15. 


\section{Appendix A: Mapping Table}

This table summarizes the BEopt inputs mapped to TrueHome. In some cases, only some options in a given category were mapped to TrueHome either due to engine limitations or time and budget restrictions.

Table 2. Summary of BEopt Inputs Mapped to TrueHome

\begin{tabular}{|c|c|c|c|}
\hline BEopt Category & BEopt Option & Mapped & Notes \\
\hline \multirow{5}{*}{ Garage } & Garage Slab & No & Not possible in TrueHome \\
\hline & Garage Walls & No & Not possible in TrueHome \\
\hline & Interzonal Walls & No & Not possible in TrueHome \\
\hline & Garage Lighting & No & Not possible in TrueHome \\
\hline & Garage Roof & No & Not possible in TrueHome \\
\hline \multirow{3}{*}{ Building } & Orientation & Yes & \\
\hline & Building Geometry & Yes & \\
\hline & Neighbors & No & Not possible in TrueHome \\
\hline \multirow{6}{*}{ Operation } & Heating Set Pt & Partially & $\begin{array}{l}\text { Fixed setpoints only, no } \\
\text { thermostat schedules }\end{array}$ \\
\hline & Cooling Set Pt & Partially & $\begin{array}{l}\text { Fixed setpoints only, no } \\
\text { thermostat schedules }\end{array}$ \\
\hline & Humidity Set Pt & No & \\
\hline & Heating/Cooling Season & Yes & Excluded from both engines \\
\hline & Occupancy & Yes & \\
\hline & Vacations & Yes & Excluded from both engines \\
\hline \multirow{7}{*}{ Walls } & Wood Stud & Yes & \\
\hline & Double Wood Stud & No & Excluded for simplicity \\
\hline & $\mathrm{CMU}$ & No & Excluded for simplicity \\
\hline & SIP & No & Excluded for simplicity \\
\hline & ICF & No & Excluded for simplicity \\
\hline & Other & No & Excluded for simplicity \\
\hline & Exterior Finish & Yes & \\
\hline \multirow{5}{*}{ Ceilings/Roofs } & Finished Roof & Yes & \\
\hline & Unfinished Attic & Yes & $\begin{array}{l}\text { Modeled in TrueHome through } \\
\text { workarounds }\end{array}$ \\
\hline & Finished Attic & No & Not possible in TrueHome \\
\hline & Roofing Material & Yes & \\
\hline & Radiant Barrier & No & Not possible in TrueHome \\
\hline
\end{tabular}




\begin{tabular}{|c|c|c|c|}
\hline BEopt Category & BEopt Option & Mapped & Notes \\
\hline \multirow{6}{*}{ Foundation/Floors } & Slab & Partially & $\begin{array}{l}\text { Only uninsulated and whole } \\
\text { slab insulation options }\end{array}$ \\
\hline & Crawlspace Construction & Partially & $\begin{array}{c}\text { Workaround constructions have } \\
\text { been implemented }\end{array}$ \\
\hline & $\begin{array}{l}\text { Finished Basement } \\
\text { Construction }\end{array}$ & No & $\begin{array}{l}\text { Additional finished zones not } \\
\text { possible in TrueHome }\end{array}$ \\
\hline & $\begin{array}{l}\text { Unfinished Basement } \\
\text { Construction }\end{array}$ & Partially & $\begin{array}{c}\text { Workaround constructions have } \\
\text { been implemented }\end{array}$ \\
\hline & Pier \& Beam Floor & No & Excluded for simplicity \\
\hline & Interzonal Floors & No & \\
\hline \multirow{5}{*}{ Thermal Mass } & Floor Mass & Yes & \\
\hline & Exterior Wall Mass & Yes & \\
\hline & $\begin{array}{l}\text { Partition Wall Thermal } \\
\text { Mass }\end{array}$ & Yes & \\
\hline & Ceiling Mass & Yes & \\
\hline & Furniture Thermal Mass & Yes & \\
\hline \multirow{7}{*}{ Windows \& Doors } & Window Areas & Yes & \\
\hline & Windows & Yes & \\
\hline & Shading & No & Not possible in TrueHome \\
\hline & Doors & No & Not possible in TrueHome \\
\hline & Door Area & No & Not possible in TrueHome \\
\hline & Eaves & No & Not possible in TrueHome \\
\hline & Overhangs & Yes & \\
\hline \multirow{3}{*}{ Airflow } & Air Leakage & Partially & Constant ACH options only \\
\hline & Natural Ventilation & No & Not possible in TrueHome \\
\hline & Mechanical Ventilation & No & Not possible in TrueHome \\
\hline \multirow{5}{*}{ Appliances } & Refrigerator & Yes & \\
\hline & Cooking Range & Yes & \\
\hline & Dishwasher & Yes & \\
\hline & Clothes Washer & Yes & \\
\hline & Clothes Dryer & Yes & \\
\hline
\end{tabular}




\begin{tabular}{|c|c|c|c|}
\hline BEopt Category & BEopt Option & Mapped & Notes \\
\hline \multirow{12}{*}{ Miscellaneous } & Misc HW loads & No & Excluded for simplicity \\
\hline & Plug Loads & Yes & \\
\hline & Extra Refrigerator & No & Excluded for simplicity \\
\hline & Freezer & No & Excluded for simplicity \\
\hline & Pool Heater & No & Excluded for simplicity \\
\hline & Pool Pump & No & Excluded for simplicity \\
\hline & Hot Tub/Spa Heater & No & Excluded for simplicity \\
\hline & Hot Tub/Spa Pump & No & Excluded for simplicity \\
\hline & Well Pump & No & Excluded for simplicity \\
\hline & Gas Fireplace & No & Excluded for simplicity \\
\hline & Gas Grill & No & Excluded for simplicity \\
\hline & Gas Lighting & No & Excluded for simplicity \\
\hline Lighting & Lighting & Yes & \\
\hline \multirow{12}{*}{$\begin{array}{c}\text { Space } \\
\text { Conditioning }\end{array}$} & System Sizing & Partially & $\begin{array}{c}\text { Using Manual } \mathrm{J} \text { for EnergyPlus, } \\
\text { built in autosizing for } \\
\text { TrueHome }\end{array}$ \\
\hline & Central AC & Partially & Single speed options only \\
\hline & Room AC & Yes & \\
\hline & Furnace & Yes & $\begin{array}{l}\text { Higher efficiency models } \\
\text { cannot be run in TrueHome }\end{array}$ \\
\hline & Minisplit & No & Excluded for simplicity \\
\hline & Boiler & Yes & $\begin{array}{l}\text { Higher efficiency models } \\
\text { cannot be run in TrueHome }\end{array}$ \\
\hline & Electric Baseboard & Yes & \\
\hline & ASHP & Partially & Single speed options only \\
\hline & GSHP & No & Excluded for simplicity \\
\hline & Ducts & No & Not possible in TrueHome \\
\hline & Ceiling Fan & No & Excluded for simplicity \\
\hline & Dehumidifier & No & Not possible in TrueHome \\
\hline
\end{tabular}




\begin{tabular}{|c|c|c|c|}
\hline BEopt Category & BEopt Option & Mapped & Notes \\
\hline \multirow{12}{*}{ Water Heating } & Mains Water Temperature & Yes & \\
\hline & Draw Profile & Partially & $\begin{array}{l}\text { Uses the same daily draw } \\
\text { volume but different profiles }\end{array}$ \\
\hline & Gas Storage & Yes & $\begin{array}{l}\text { Higher efficiency models } \\
\text { cannot be run in TBM }\end{array}$ \\
\hline & Electric Storage & Yes & \\
\hline & Oil Storage & Yes & $\begin{array}{l}\text { Higher efficiency models } \\
\text { cannot be run in TBM }\end{array}$ \\
\hline & Gas Tankless & Yes & $\begin{array}{l}\text { Higher efficiency models } \\
\text { cannot be run in TBM }\end{array}$ \\
\hline & Electric Tankless & Yes & \\
\hline & HPWH & Yes & \\
\hline & Distribution & No & Not possible in TrueHome \\
\hline & Demand Recirculation & No & Not possible in TrueHome \\
\hline & Timer Recirculation & No & Not possible in TrueHome \\
\hline & SWH & No & Excluded for simplicity \\
\hline PV & PV & No & Excluded for simplicity \\
\hline
\end{tabular}




\section{Appendix B: BEopt TrueHome/EnergyPlus Test Suite Coverage}

This table gives the range of BEopt options analyzed in TrueHome and EnergyPlus during this work.

Table 3. BEopt TrueHome/EnergyPlus Test Suite Coverage

\begin{tabular}{|c|c|c|}
\hline BEopt Group & BEopt Category & BEopt Option \\
\hline \multirow{16}{*}{ Building } & \multirow{16}{*}{ Orientation } & North \\
\hline & & NNE \\
\hline & & Northeast \\
\hline & & ENE \\
\hline & & East \\
\hline & & ESE \\
\hline & & Southeast \\
\hline & & SSE \\
\hline & & South \\
\hline & & SSW \\
\hline & & Southwest \\
\hline & & WSW \\
\hline & & West \\
\hline & & WNW \\
\hline & & Northwest \\
\hline & & NNW \\
\hline \multirow{15}{*}{ Operation } & \multirow{8}{*}{ Heating Set Point } & $68^{\circ} \mathrm{F}$ \\
\hline & & $69^{\circ} \mathrm{F}$ \\
\hline & & $70^{\circ} \mathrm{F}$ \\
\hline & & $71^{\circ} \mathrm{F}$ \\
\hline & & $72^{\circ} \mathrm{F}$ \\
\hline & & $73^{\circ} \mathrm{F}$ \\
\hline & & $74^{\circ} \mathrm{F}$ \\
\hline & & $75^{\circ} \mathrm{F}$ \\
\hline & \multirow{7}{*}{ Cooling Set Point } & $73^{\circ} \mathrm{F}$ \\
\hline & & $74^{\circ} \mathrm{F}$ \\
\hline & & $75^{\circ} \mathrm{F}$ \\
\hline & & $76^{\circ} \mathrm{F}$ \\
\hline & & $77^{\circ} \mathrm{F}$ \\
\hline & & $78^{\circ} \mathrm{F}$ \\
\hline & & $79^{\circ} \mathrm{F}$ \\
\hline
\end{tabular}




\begin{tabular}{|c|c|c|}
\hline BEopt Group & BEopt Category & BEopt Option \\
\hline & & $80^{\circ} \mathrm{F}$ \\
\hline \multirow{23}{*}{ Walls } & \multirow{10}{*}{ Wood Stud } & None \\
\hline & & Uninsulated, $2 \times 4,16$ in. o.c. \\
\hline & & R-11 Fiberglass Batt, Gr-1, $2 \times 4,16$ in. o.c. \\
\hline & & R-13 Fiberglass Batt, Gr-1, $2 \times 4,16$ in. o.c. \\
\hline & & R-19 Fiberglass Batt, Gr-1, $2 \times 6,24$ in. o.c. \\
\hline & & R-21 Fiberglass Batt, Gr-1, $2 \times 6,24$ in. o.c. \\
\hline & & R-13 Cellulose, Gr-1, $2 \times 4,16$ in. o.c. \\
\hline & & R-19 Cellulose, Gr-1, $2 \times 6,24$ in. o.c. \\
\hline & & R-13 Fiberglass, Gr-1, $2 \times 4,16$ in. o.c. \\
\hline & & R-19 Fiberglass, Gr-1, $2 \times 6,24$ in. o.c. \\
\hline & \multirow{2}{*}{ Wall Sheathing } & None \\
\hline & & R-5 XPS \\
\hline & \multirow{11}{*}{ Exterior Finish } & Stucco, Medium/Dark \\
\hline & & Brick, Light \\
\hline & & Brick, Medium/Dark \\
\hline & & Wood, Light \\
\hline & & Wood, Medium/Dark \\
\hline & & Aluminum, Light \\
\hline & & Aluminum, Medium/Dark \\
\hline & & Vinyl, Light \\
\hline & & Vinyl, Medium/Dark \\
\hline & & Fiber-Cement, Light \\
\hline & & Fiber-Cement, Medium/Dark \\
\hline \multirow{13}{*}{ Ceilings/Roofs } & \multirow{13}{*}{ Unfinished Attic } & Uninsulated, Vented \\
\hline & & Ceiling R-11 Fiberglass, Vented \\
\hline & & Ceiling R-19 Fiberglass, Vented \\
\hline & & Ceiling R-30 Fiberglass, Vented \\
\hline & & Ceiling R-38 Fiberglass, Vented \\
\hline & & Ceiling R-49 Fiberglass, Vented \\
\hline & & Ceiling R-11 Cellulose, Vented \\
\hline & & Ceiling R-19 Cellulose, Vented \\
\hline & & Ceiling R-30 Cellulose, Vented \\
\hline & & Ceiling R-38 Cellulose, Vented \\
\hline & & Ceiling R-49 Cellulose, Vented \\
\hline & & Ceiling R-30 Fiberglass Batt, Vented \\
\hline & & Ceiling R-38 Fiberglass Batt, Vented \\
\hline
\end{tabular}




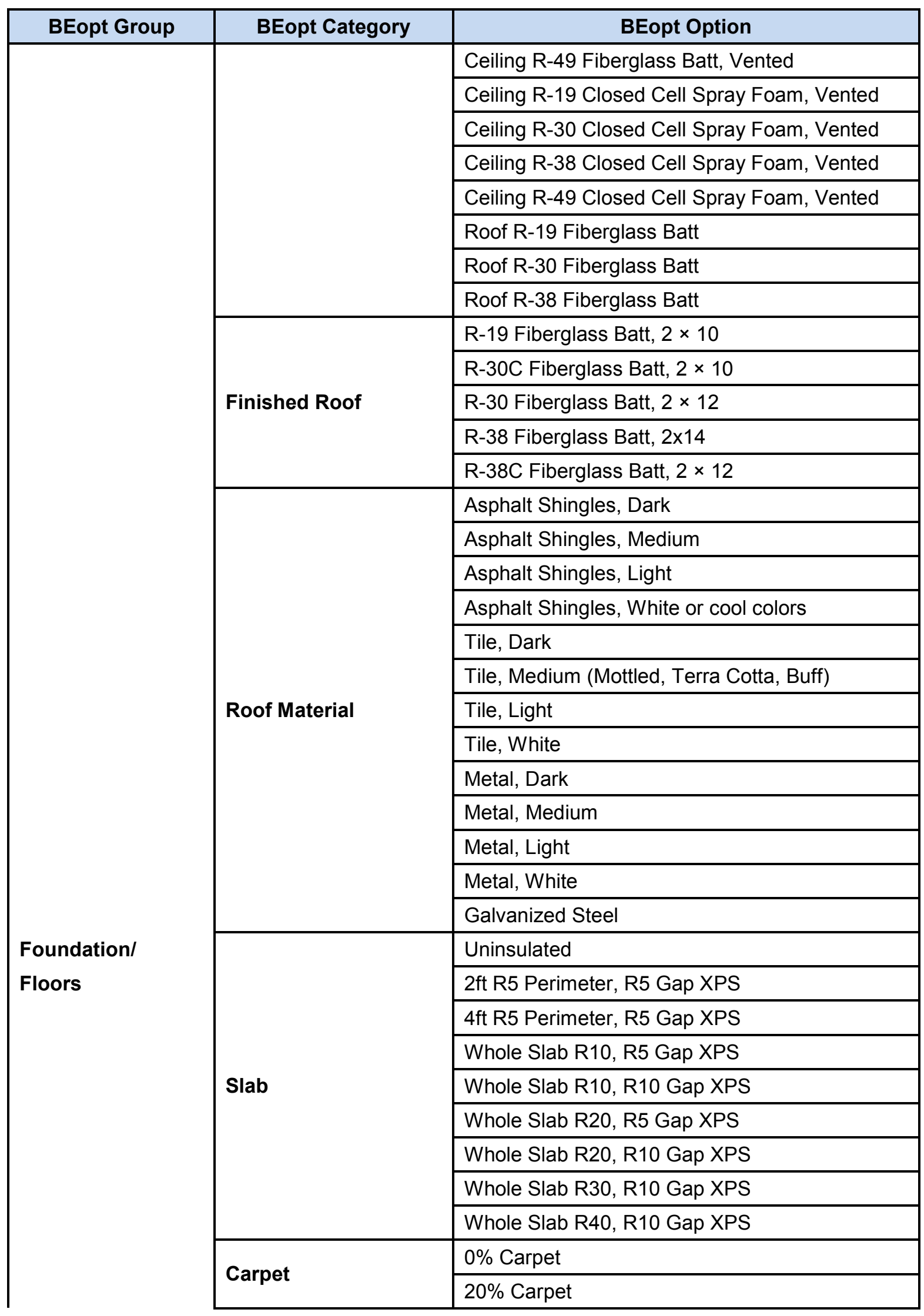




\begin{tabular}{|c|c|c|}
\hline BEopt Group & BEopt Category & BEopt Option \\
\hline & & 40\% Carpet \\
\hline & & $60 \%$ Carpet \\
\hline & & $80 \%$ Carpet \\
\hline & & $100 \%$ Carpet \\
\hline \multirow{3}{*}{ Thermal Mass } & \multirow{3}{*}{ Floor Mass } & None \\
\hline & & Wood Surface \\
\hline & & 2 in. Gypsum Concrete \\
\hline \multirow{26}{*}{$\begin{array}{l}\text { Windows and } \\
\text { Doors }\end{array}$} & \multirow{11}{*}{ Window Areas } & None \\
\hline & & 18.0\% F25 B25 L25 R25 \\
\hline & & 18.0\% F20 B40 L20 R20 \\
\hline & & $15.0 \%$ F25 B25 L25 R25 \\
\hline & & $15.0 \%$ F20 B40 L20 R20 \\
\hline & & 12.0\% F25 B25 L25 R25 \\
\hline & & $12.0 \%$ F20 B40 L20 R20 \\
\hline & & 15.0\% F33 B33 L0 R33 (attached L) \\
\hline & & 15.0\% F33 B33 L33 R0 (attached R) \\
\hline & & 15.0\% F50 B50 L0 R0 (attached L,R) \\
\hline & & $50 \mathrm{ft}^{2}$, all facades \\
\hline & \multirow{15}{*}{ Windows } & 1-Pane, Clear, Metal Frame \\
\hline & & 1-Pane, Clear, Non-metal Frame \\
\hline & & 2-Pane, Clear, Metal Frame, Air Fill \\
\hline & & $\begin{array}{l}\text { 2-Pane, Clear, Metal w/ Thermal Break Frame, } \\
\text { Air Fill }\end{array}$ \\
\hline & & 2-Pane, Clear, Non-metal Frame, Air Fill \\
\hline & & $\begin{array}{l}\text { 2-Pane, High-Gain Low-E, Non-metal Frame, Air } \\
\text { Fill }\end{array}$ \\
\hline & & $\begin{array}{l}\text { 2-Pane, Medium-Gain Low-E, Non-metal Frame, } \\
\text { Air Fill }\end{array}$ \\
\hline & & $\begin{array}{l}\text { 2-Pane, Low-Gain Low-E, Non-metal Frame, Air } \\
\text { Fill }\end{array}$ \\
\hline & & $\begin{array}{l}\text { 2-Pane, High-Gain Low-E, Non-metal Frame, } \\
\text { Argon Fill }\end{array}$ \\
\hline & & $\begin{array}{l}\text { 2-Pane, Med-Gain Low-E, Non-metal Frame, } \\
\text { Argon Fill }\end{array}$ \\
\hline & & $\begin{array}{l}\text { 2-Pane, Low-Gain Low-E, Non-metal Frame, } \\
\text { Argon Fill }\end{array}$ \\
\hline & & $\begin{array}{l}\text { 2-Pane, High-Gain Low-E, Insulated Frame, Air } \\
\text { Fill }\end{array}$ \\
\hline & & $\begin{array}{l}\text { 2-Pane, Med-Gain Low-E, Insulated Frame, Air } \\
\text { Fill }\end{array}$ \\
\hline & & $\begin{array}{l}\text { 2-Pane, Low-Gain Low-E, Insulated Frame, Air } \\
\text { Fill }\end{array}$ \\
\hline & & 2-Pane, High-Gain Low-E, Insulated Frame, \\
\hline
\end{tabular}




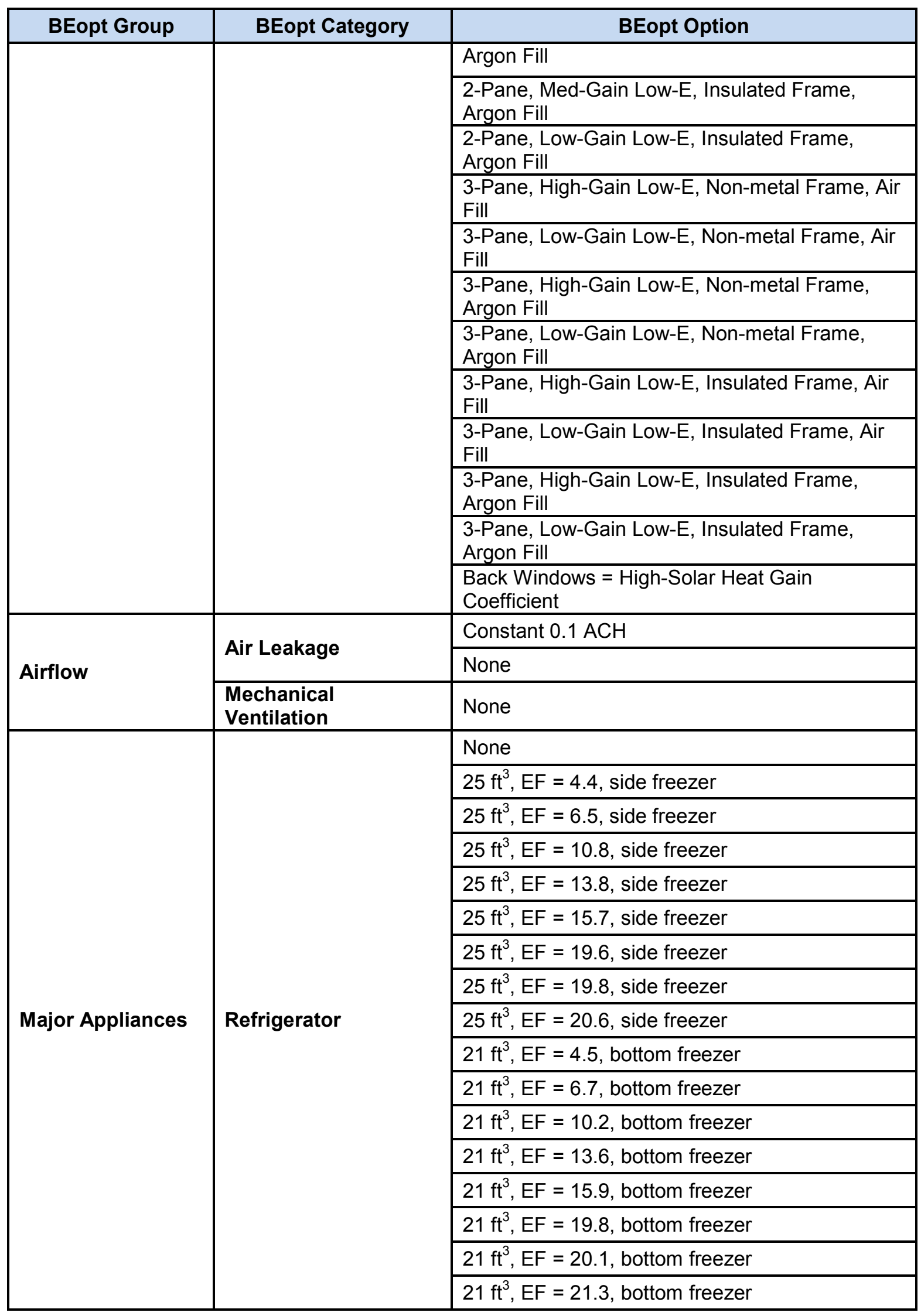




\begin{tabular}{|c|c|c|}
\hline BEopt Group & BEopt Category & BEopt Option \\
\hline & & $18 \mathrm{ft}^{3}, \mathrm{EF}=4.4$, top freezer \\
\hline & & $18 \mathrm{ft}^{3}, \mathrm{EF}=6.9$, top freezer \\
\hline & & $18 \mathrm{ft}^{3}, \mathrm{EF}=10.5$, top freezer \\
\hline & & $18 \mathrm{ft}^{3}, \mathrm{EF}=14.1$, top freezer \\
\hline & & $18 \mathrm{ft}^{3}, \mathrm{EF}=15.9$, top freezer \\
\hline & & $18 \mathrm{ft}^{3}, \mathrm{EF}=19.9$, top freezer \\
\hline & & $18 \mathrm{ft}^{3}, \mathrm{EF}=20.4$, top freezer \\
\hline & & $18 \mathrm{ft}^{3}, \mathrm{EF}=21.9$, top freezer \\
\hline & \multirow{4}{*}{ Cooking Range } & None \\
\hline & & Electric \\
\hline & & Electric, Induction \\
\hline & & Gas, Conventional \\
\hline & \multirow{3}{*}{ Dishwasher } & None \\
\hline & & 318 Annual kWh \\
\hline & & 290 Annual kWh \\
\hline & \multirow{5}{*}{ Clothes Washer } & None \\
\hline & & Standard \\
\hline & & ENERGY STAR \\
\hline & & Standard - Cold Only \\
\hline & & ENERGY STAR - Cold Only \\
\hline & \multirow{3}{*}{ Clothes Dryer } & None \\
\hline & & Electric \\
\hline & & Gas \\
\hline \multirow{8}{*}{ Miscellaneous } & \multirow{8}{*}{ Other Electric Loads } & None \\
\hline & & 4 \\
\hline & & 2 \\
\hline & & 1.5 \\
\hline & & 1 \\
\hline & & 0.75 \\
\hline & & 0.5 \\
\hline & & 0.25 \\
\hline \multirow{6}{*}{ Lighting } & \multirow{6}{*}{ Lighting } & None \\
\hline & & Benchmark \\
\hline & & $20 \%$ Fluorescent, Hardwired \\
\hline & & $40 \%$ Fluorescent, Hardwired \\
\hline & & $60 \%$ Fluorescent, Hardwired \\
\hline & & $80 \%$ Fluorescent, Hardwired \\
\hline
\end{tabular}




\begin{tabular}{|c|c|c|}
\hline BEopt Group & BEopt Category & BEopt Option \\
\hline & & $100 \%$ Fluorescent, Hardwired \\
\hline & & $20 \%$ Fluorescent, Hardwired and Plugin \\
\hline & & $40 \%$ Fluorescent, Hardwired and Plugin \\
\hline & & $60 \%$ Fluorescent, Hardwired and Plugin \\
\hline & & $80 \%$ Fluorescent, Hardwired and Plugin \\
\hline & & $100 \%$ Fluorescent, Hardwired and Plugin \\
\hline & & $\begin{array}{l}50 \% \text { Fluorescent, } 10 \% \text { LED, Hardwired and } \\
\text { Plugin }\end{array}$ \\
\hline & & $1,300 \mathrm{kWh}$ \\
\hline \multirow{27}{*}{$\begin{array}{l}\text { Space } \\
\text { Conditioning }\end{array}$} & \multirow{12}{*}{$\begin{array}{l}\text { Central Air } \\
\text { Conditioner }\end{array}$} & None \\
\hline & & SEER 8 \\
\hline & & SEER 10 \\
\hline & & SEER 13 \\
\hline & & SEER 14 \\
\hline & & SEER 15 \\
\hline & & SEER 16 \\
\hline & & SEER 16 (2 Stage) \\
\hline & & SEER 17 \\
\hline & & SEER 18 \\
\hline & & SEER 21 \\
\hline & & SEER 24.5 \\
\hline & \multirow{9}{*}{ Furnace } & None \\
\hline & & Electric, $100 \%$ AFUE \\
\hline & & Gas, $60 \%$ AFUE \\
\hline & & Gas, $64 \%$ AFUE \\
\hline & & Gas, $68 \%$ AFUE \\
\hline & & Gas, $72 \%$ AFUE \\
\hline & & Gas, $76 \%$ AFUE \\
\hline & & Gas, $78 \%$ AFUE \\
\hline & & Gas, $80 \%$ AFUE \\
\hline & \multirow{4}{*}{ Boiler } & None \\
\hline & & Gas, Hot Water, Forced Draft, $72 \%$ AFUE \\
\hline & & Gas, Hot Water, Forced Draft, 76\% AFUE \\
\hline & & Gas, Hot Water, Forced Draft, $80 \%$ AFUE \\
\hline & \multirow{2}{*}{ Electric Baseboard } & None \\
\hline & & 100\% Efficiency \\
\hline \multirow{2}{*}{ Water Heating } & \multirow{2}{*}{ Water Heater } & None \\
\hline & & Electric Standard \\
\hline
\end{tabular}




\begin{tabular}{|c|c|c|}
\hline BEopt Group & BEopt Category & BEopt Option \\
\hline & & Electric Premium \\
\hline & & Electric Tankless \\
\hline & & Gas Standard \\
\hline & & Gas Tankless \\
\hline & & Propane Standard \\
\hline & & $\mathrm{HPWH}, 50 \mathrm{gal}$ \\
\hline & & $\mathrm{HPWH}, 50 \mathrm{gal}, 140^{\circ} \mathrm{F}$ \\
\hline & & HPWH, 50 gal, Exhaust Ducting \\
\hline & & HPWH, 50 gal, In Confined Space \\
\hline & & $\mathrm{HPWH}, 80 \mathrm{gal}$ \\
\hline
\end{tabular}

